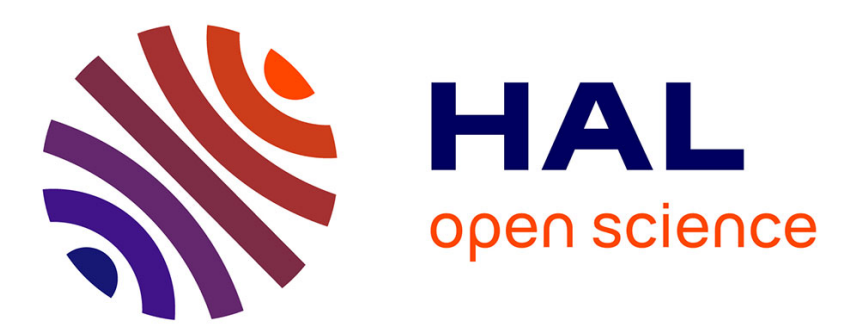

\title{
Vers une agriculture sans ruralité ? La renégociation de la place de l'agriculture sur l'île de Bréhat (xxe-xxie siècle)
}

\author{
Sandrine Dupé, Jérôme Cardinal, Alix Levain
}

\section{- To cite this version:}

Sandrine Dupé, Jérôme Cardinal, Alix Levain. Vers une agriculture sans ruralité ? La renégociation de la place de l'agriculture sur l'île de Bréhat (xxe-xxie siècle). Norois, 2021, 259-260, pp.35-52. 10.4000/norois.10922 . hal-03502843

\section{HAL Id: hal-03502843 \\ https://hal.science/hal-03502843}

Submitted on 26 Dec 2021

HAL is a multi-disciplinary open access archive for the deposit and dissemination of scientific research documents, whether they are published or not. The documents may come from teaching and research institutions in France or abroad, or from public or private research centers.
L'archive ouverte pluridisciplinaire HAL, est destinée au dépôt et à la diffusion de documents scientifiques de niveau recherche, publiés ou non, émanant des établissements d'enseignement et de recherche français ou étrangers, des laboratoires publics ou privés. 
VERS UNE AGRICULTURE SANS RURALITÉ ? LA RENÉGOCIATION DE LA PLACE DE L'AGRICULTURE SUR L'ÎLE DE BRÉHAT (XX ${ }^{\mathrm{E}}-\mathrm{XXI}^{\mathrm{E}}$ SIĖCLE)

Sandrine Dupé, Jérôme Cardinal, Alix Levain

Presses universitaires de Rennes | "Norois »

2021/2 n²59-260 | pages 35 à 52

ISSN 0029-182X

ISBN 9782753585881

DOI 10.4000/norois.10922

Article disponible en ligne à l'adresse :

https://www.cairn.info/revue-norois-2021-2-page-35.htm

Distribution électronique Cairn.info pour Presses universitaires de Rennes.

(C) Presses universitaires de Rennes. Tous droits réservés pour tous pays.

La reproduction ou représentation de cet article, notamment par photocopie, n'est autorisée que dans les limites des conditions générales d'utilisation du site ou, le cas échéant, des conditions générales de la licence souscrite par votre établissement. Toute autre reproduction ou représentation, en tout ou partie, sous quelque forme et de quelque manière que ce soit, est interdite sauf accord préalable et écrit de l'éditeur, en dehors des cas prévus par la législation en vigueur en France. Il est précisé que son stockage dans une base de données est également interdit. 


\section{Norois}

Environnement, aménagement, société

\section{Vers une agriculture sans ruralité ? La renégociation de la place de l'agriculture sur l'île de Bréhat ( $\mathrm{XX}^{\mathrm{e}}-\mathrm{XXI}^{\mathrm{e}}$ siècle)}

Agriculture besides rurality? Renegociating the place of farming activities on Bréhat Island ( $20^{\text {th }}-21^{\text {st }}$ century)

\section{Sandrine Dupé, Jérôme Cardinal et Alix Levain}

\section{OpenEdition}

\section{Journals}

Édition électronique

URL : https://journals.openedition.org/norois/10922

DOI : 10.4000/norois. 10922

ISSN : 1760-8546

\section{Éditeur}

Presses universitaires de Rennes

\section{Édition imprimée}

Date de publication : 15 décembre 2021

Pagination : 35-52

ISBN : 978-2-7535-8588-1

ISSN : 0029-182X

Distribution électronique Cairn

\section{CAIRN}

CHERCHER, REPÉRER, AVANCER

\section{Référence électronique}

Sandrine Dupé, Jérôme Cardinal et Alix Levain, « Vers une agriculture sans ruralité ? La renégociation de la place de l'agriculture sur l'île de Bréhat ( $x x^{\mathrm{e}}$-xx| ${ }^{\mathrm{e}}$ siècle) », Norois [En ligne], 259-260 | 2021, mis en ligne le 04 janvier 2024, consulté le 02 décembre 2021. URL : http://journals.openedition.org/norois/ 10922 ; DOI : https://doi.org/10.4000/norois.10922 

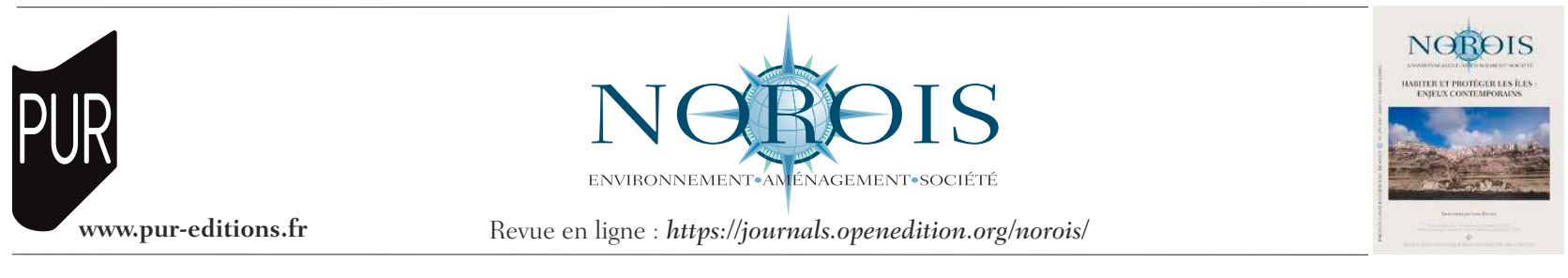

\title{
Vers une agriculture sans ruralité? La renégociation de la place de l'agriculture sur l'île de Bréhat $\left(\mathrm{xx}^{\mathrm{e}}-\mathrm{XXI}^{\mathrm{e}}\right.$ siècle)
}

\author{
Agriculture besides rurality? Renegociating the place of farming activities on Bréhat Island \\ $\left(20^{\text {th }}-21^{\text {st }}\right.$ century)
}

\author{
Sandrine Dupéa, Jérôme CARDinal ${ }^{b}$ et Alix Levain ${ }^{c}$
}

\begin{abstract}
a Auteure correspondante, Laboratoire d'éco-anthropologie, UMR 7206, Muséum national d'histoire naturelle, site du Musée de l'Homme, 17 place du Trocadéro, 75116 Paris, France (sandrinedupe@gmail.com)

${ }^{\text {b } C i t e ́ s, ~ T e r r i t o i r e s, ~ E n v i r o n n e m e n t ~ e t ~ S o c i e ́ t e ́ s ~(C I T E R E S), ~ U M R ~ 7324, ~ E ́ q u i p e ~ D y n a m i q u e ~ e t ~ A c t i o n ~ T e r r i t o r i a l e s ~}$ et Environnementales (DATE), 35 Allée Ferdinand de Lesseps, 37200 Tours, France (cardinal.jeromel@gmail.com)

c CNRS, Laboratoire Aménagement des Usages des Ressources et des Espaces marins et littoraux, UMR 6308, AMURE, Institut universitaire européen de la mer, Rue Dumont d'Urville, 29280 Plouzané, France (alix.levain@univ-brest.fr)
\end{abstract}

Résumé : Cet article analyse les effets de la littoralité sur l'évolution de l'inscription socio-spatiale de l'activité agricole, quand elle est exacerbée par le contexte insulaire, comme sur l'île de Bréhat. En croisant analyse spatiale et données ethnographiques, nous y repérons et décrivons, depuis le $\mathrm{xx}_{\mathrm{e}}^{\mathrm{e}}$ siècle, des moments de déstabilisations successives construits à plusieurs échelles, qui redessinent la place et les fonctions de l'agriculture au cours du temps. Trois agencements socio-spatiaux temporairement stables, que nous qualifierons de "pactes territoriaux », émergent. Les évolutions de l'inscription de l'agriculture dans l'espace social local sont corrélées à une transformation de la ruralité sur le territoire. Le premier pacte territorial s'étend du début du xx siècle jusqu'aux années 1960. L'agriculture est vivrière et constitue un socle dans l'organisation sociale de l'île, bien que l'arrivée de touristes diversifie les ressources économiques locales. Au tournant des années 1960, l'ampleur du tourisme et la difficulté à s’inscrire dans les nouvelles politiques agricoles nationales déstabilisent cet équilibre. L'agriculture se marginalise et perd sa fonction localement structurante : la fin des paysans coïncide avec la fin de la ruralité. Enfin, l'activité agricole se redynamise au cours de la dernière décennie, en même temps qu'elle est mise en débat publiquement, re-créant un espace de proximité entre divers usagers du territoire. C'est une forme de ruralité ré-inventée qui est consacrée, ouvrant une position circonscrite aux acteurs agricoles. Pour prétendre obtenir du foncier disponible, ils doivent s'insérer dans un modèle d'agriculture multifonctionnelle, faisant de l'île un jardin aux fonctions productives et esthétiques. Les rapports de force et la distribution du pouvoir préexistant évoluent peu, et l'agriculture locale reste marquée par des formes de précarité économique et foncière.

Abstract: This article analyzes the effects of the coastline on the evolution of the socio-spatial insertion of agricultural activity, when it
is exacerbated by the insular context, as on the island of Brehat. By combining spatial analysis and ethnographic data, we describe the
forms and effects of the successive destabilizations built on several scales that Brehat has known since the $20^{\text {th }}$ century. We analyze how
they redesign the place and functions of agriculture over time. Three temporarily stable socio-spatial arrangements, which we qualify as
"territorial pacts" emerge. Changes in the inclusion of agriculture in the local social space are correlated with a transformation of rurality
in the territory. The first territorial pact extends from the beginning of the $20^{\text {th }}$ century until the 1960 s. Subsistence farming constitutes
a base in the social organization of the island, although the arrival of tourists diversifies the local economic resources. At the turn of the
1960 s, the extent of tourism and the difficulty of fitting into new national agricultural policies destabilizes this balance. Farming is mar-
ginalized and loses its structuring function locally: the vanishing of peasants coincides with the end of rurality. Finally, farming activity
has gained momentum over the past decade, at the same time as it is the subject of public debate, recreating a space of proximity between 
various stakeholders. It is a form of re-invented rurality that is consecrated, opening up a circumscribed position to agricultural actors. To claim to obtain available land, they must fit in a multifunctional agricultural model, making the island a garden with productive and aesthetic functions. The pre-existing balance of power changes little, and local farming remains characterized by forms of economic and land insecurity.

Mots clés : agriculture - ruralité - tourisme - ethnographie - Bréhat - île

Keywords: agriculture - rurality - tourism - ethnography - Bréhat - island

Nous remercions l'ensemble des personnes qui ont contribué à cette enquête : celles qui nous ont apporté leur témoignage, l'équipe de recherche du projet Paroles et chemins de l'agriculture littorale et Mélanie Congrétel pour avoir enrichi ce travail de leur point de vue, ainsi que la Fondation de France, la Région Bretagne, l'Inrae et Ireps Bretagne, qui l'ont financée et portée.

\section{INTRODUCTION}

Les activités agricoles connaissent, dans les îles de la façade Atlantique comme dans de nombreux espaces littoraux de France métropolitaine, des transformations rapides dont la nature et l'ampleur restent largement à documenter, mais qui sont marquées par plusieurs phénomènes qui entrent régulièrement en tension.

La compréhension de la spécificité des dynamiques agro-littorales ne saurait ainsi se réduire à la mise en avant de singularités locales et de productions emblématiques labellisées. Ces dynamiques résultent davantage de frictions entre des phénomènes globaux - comme la modernisation agricole et la massification du tourisme - et une organisation sociale locale spécifique, au sein d'un espace social localisé (Laferté, 2014) dont le caractère rural mérite d'être questionné. À la différence de ce qui peut être observé dans certains espaces ruraux marqués par une correspondance entre isolement géographique et marginalisation sociale des populations, les territoires littoraux connaissent en effet des formes complexes d'organisation sociale et territoriale dans des espaces à la fois circonscrits et attractifs. La configuration insulaire accentue le brouillage des catégories de l'urbain et du rural, du fait de l'attractivité et de la patrimonialisation des littoraux, complexifiant la compréhension des trajectoires de l'agriculture. Ce brouillage est renforcé par la rupture apparente que cette activité historiquement structurante a connue à l'occasion du décrochage des années 1950 et 1960, période où s’accélère sur le continent la modernisation agricole. L'espace n'a plus seulement une fonction productive, mais également contemplative et patrimoniale. Certaines franges du littoral breton, et a fortiori certaines îles, sont restées largement à l'écart de la modernisation agricole, jusqu'à connaître une quasi-disparition de l'activité agricole. En tant que territoire attractif pour le tourisme (Huchet du Guermeur, 2000), Bréhat, sur laquelle porte cette recherche (figure 1), constitue à ce titre un cas d'école. Sous quelles formes et à quelles conditions l'agriculture a-t-elle pu y subsister?

Pour rendre compte des transformations de la place des activités agricoles sur Bréhat, nous aborderons ici le phénomène insulaire comme un processus socio-historique structurant un ensemble de cadres matériels et immatériels déterminants pour ces activités. L'hétérogénéité de ces cadres engage à considérer l'agriculture comme une activité sociale, entendue comme la synthèse de trois dimensions complémentaires définies par H. Arendt (1983), à savoir le travail, ou la vie économique, l'œuvre, qui relève de l'accomplissement personnel des habitants, et l'action, ou la vie politique et associative. Cette définition inclusive intègre aussi bien les pratiques agricoles vivrières, qu'une activité économique professionnelle, ou un ensemble de pratiques hétérogènes de production et de transformation de ressources primaires. Adopter une vision holistique de ce type permet également de mettre en avant la dimension transactionnelle de l'activité agricole, dans le sens où celle-ci « produit et reproduit des relations particulières avec les sociétés, les individus et les communautés »(Stoessel-Ritz, 2017 : 153), adossées à des relations aux milieux naturels et aux communautés non-humaines. Les formes de présence de l'agriculture contribuent ainsi à modeler et à révéler des contrats sociaux souvent implicites qui relient, sur un territoire donné, la transformation des milieux à des fins productives aux habitants, aux citoyens, aux consommateurs. 
Figure 1 : île de Bréhat, constituée de deux îles principales reliées par un isthme (île Nord et île Sud), insérées dans un archipel, et situation sur la façade Atlantique

Bréhat, composed of two main islands (Northern island and Southern island) connected by an isthmus; situation of the archipelago on the Atlantic coast

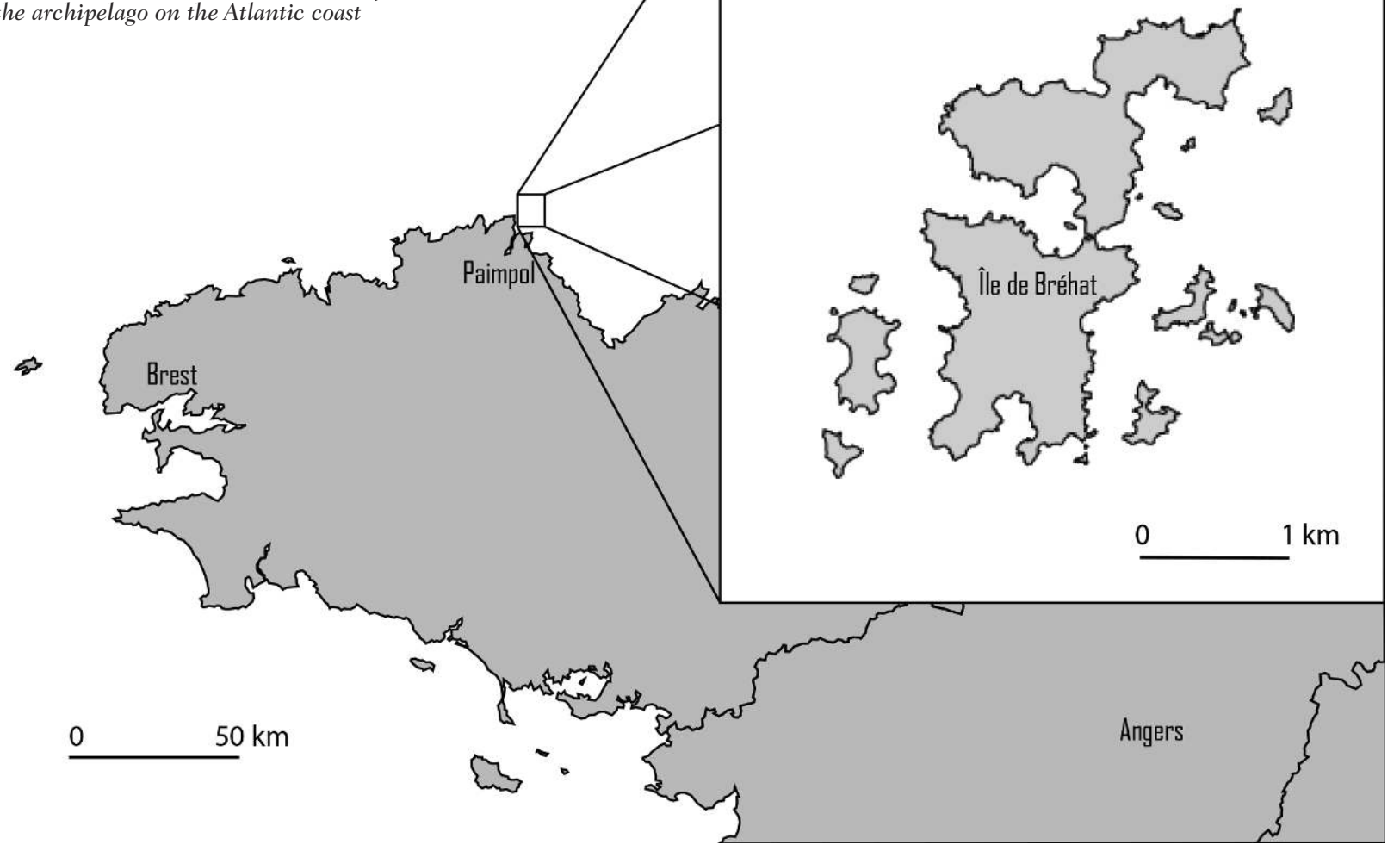

Dans cette perspective, la ruralité ne peut s'appréhender uniquement par l'objectivation spatiale (la faible densité, la présence de terres exploitées) mais doit bien plutôt se concevoir comme une forme de socialité de proximité spécifique, issue de négociations portant sur l'articulation entre différentes activités productives et de représentations dominantes du territoire et de son développement. L'analyse des effets de l'industrialisation de l'agriculture sur les sociabilités locales dans les campagnes (Mendras, 1967; Prével, 2008), en particulier dans le contexte breton (Canévet, 1980, 1992) a largement mis en évidence le processus de dissociation entre agriculture et ruralité. De façon plus récente, en contexte urbain, le réinvestissement de la ville par la production alimentaire conduit à penser de nouvelles formes de ruralité, marquées par un processus précaire de reconquête (Granchamp, 2017).

Il s'agit alors de ne pas présager d'un cadre d'analyse urbain, littoral ou rural pour appréhender l'évolution de la ruralité à Bréhat, mais d'observer l'évolution de la place de l'agriculture dans le temps, comme produit des frictions entre des dynamiques locales et des transformations plus globales.

La configuration bréhatine se situe, nous le montrerons dans cet article, au croisement de ces différents processus de déconstruction-reconstruction de la ruralité. Nous y décrirons les formes et les effets des déstabilisations successives qu'a connues Bréhat et la façon dont elles contribuent à y redessiner la place et les fonctions de l'agriculture, en croisant analyse spatiale et données ethnographiques.

Nous considèrerons ainsi, au travers des transformations de l'activité agricole, les multiples façons dont l'espace social localisé bréhatin s'attache et se distingue de celui du continent au cours du temps. Nous caractériserons sur cette base différents « pactes territoriaux » (Santos, 2000; Albaladejo, 2012) qui ont successivement défini, régulé et contribué à reconfigurer sur le plan social, technique et politique, la place et le sens de l'activité agricole à Bréhat, en relation avec les autres activités insulaires. Nous mettrons ainsi en évidence un faisceau de contraintes et de possibles tacites ou manifestes, 
construits à différentes échelles, qui déterminent l'insertion socio-spatiale de l'activité agricole. Les résonances et frictions entre ces échelles contribuent à constituer des agencements stables rythmés par des phases de structuration, déstructuration et restructuration de l'activité agricole sur un territoire. Nous nous attacherons particulièrement à décrire dans la dernière partie de cet article les conditions dans lesquelles ce pacte se renégocie au tournant des années 2010. Ces années sont marquées par la réimplantation d'activités agricoles diversifiées, inscrites dans l'économie saisonnière et touristique, et par un renouvellement des conditions et des enjeux de la mise en débat de l'agriculture dans l'espace public. Ces dynamiques, récentes et fragiles, questionnent la réinscription de l'agriculture dans une forme renégociée de ruralité.

\section{DES TÉMOIGNAGES ÉCRITS ET VISUELS AUX DISCOURS CONTEMPORAINS SUR L'ACTIVITÉ AGRICOLE}

\section{Traces de l'agriculture à Bréhat depuis le $\mathrm{XIX}^{\mathrm{e}}$ siècle}

À Bréhat, le parcellaire très morcelé et le nombre restreint d'agriculteurs sur la commune (secret statistique) limitent la description des caractéristiques agricoles à partir des statistiques agricoles nationales. Les registres parcellaires graphiques, servant de référence à l'instruction des aides de la politique agricole commune, ne sont pas renseignés. De l'aveu d'un éleveur enquêté qui s'est attelé à cette tâche, dans l'espoir de bénéficier de subventions, le travail de recension des propriétaires fonciers rend ce travail démesuré au regard de son intérêt économique. Bien qu'incomplet, le recensement général agricole ${ }^{1}$ renseigne toutefois sur le nombre d'exploitations en 1988, 2000 et 2010 (à savoir 5 pour chacune de ces dates). La variété des cas limite, rapportée au faible nombre d'agriculteurs, rend malgré tout ces indicateurs peu signifiants. Le recours à d'autres sources est donc indispensable.

Bréhat a constitué un objet d'études universitaires, particulièrement en géographie et en histoire, au cours du xxe siècle. En 1925, G. Vergez-Tricom,

1. Le RGA inclut les statistiques sur le nombre d'exploitations, les orientations technico-économiques des communes, les surfaces agricoles utilisées, les productions et surfaces concernées, les surfaces toujours en herbe et les surfaces herbagères principales figure de l'École française de géographie, s’installe temporairement à Bréhat. Mobilisant une approche ethnographique, elle documente précisément l'activité agricole, livrant ainsi des informations précieuses sur les types de production, l'organisation de la société insulaire et les mutations socio-spatiales qui marquent le tournant du $\mathrm{xx}^{\mathrm{e}}$ siècle. L'agriculture bréhatine ne sera plus traitée de manière aussi complète jusqu'aux années 2010. Depuis une trentaine d'années, elle trouve toutefois sa place, dans une perspective comparative, dans un ensemble de travaux qui considère les îles du Ponant comme une entité analytique à part entière (Péron, 1993; Guillemet, 2000; Brigand, 2002; Buhot, 2005; Gariglietti-Brachetto, 2013). Lagriculture y est envisagée comme étant façonnée par l'insularité.

L'activité agricole apparaît également à travers des travaux d'érudits locaux. À la fin du $\mathrm{xx}^{\mathrm{e}}$ siècle, sont collectées et mises en forme de nombreuses archives témoignant de l'histoire de l'île. J.-L. et M. Le Pache (1991) dressent ainsi, pour la période révolutionnaire, un portrait de la vie locale dans lequel l'activité agricole est omniprésente, sans constituer la focale de leur récit. L'abbé Menguy, recteur à Bréhat de 1986 à 2000, l'évoque dans certaines de ses chroniques sur l'histoire locale publiées dans le bulletin paroissial (Menguy, 2005). Ces travaux se nourrissent des écrits de savants, administrateurs et voyageurs, venus à Bréhat entre le milieu du XIX ${ }^{\mathrm{e}}$ siècle et celui du Xx ${ }^{\mathrm{e}}$ siècle, comme ceux de Marteville et Varin (1843) ou de Rigaud (1890), inspecteur des écoles, qui y consacre quelques pages dans sa Géographie historique des Côtes du Nord. Les éléments qu'ils fournissent sont avant tout d'ordre administratif, même s'ils s'enrichissent de quelques observations personnelles.

Les nombreux peintres qui ont posé leur toile à Bréhat au début du $\mathrm{xx}^{\mathrm{e}}$ peignent des paysages aux végétations rases où, selon les compositions, se superposent espaces maritimes et terrestres, cultivés et habités. L'agriculture forme un contrepoint paysager discret aux scènes marines ou aux portraits d'îliennes. Ces toiles sont autant d'indices de l'organisation sociale et des types de productions agricoles de l'époque (figure 2).

À cela s'ajoutent des photographies, éditées en cartes postales (figure 3). Ce patrimoine iconographique est en partie rendu accessible par des intellectuels soucieux de donner à voir le patrimoine 


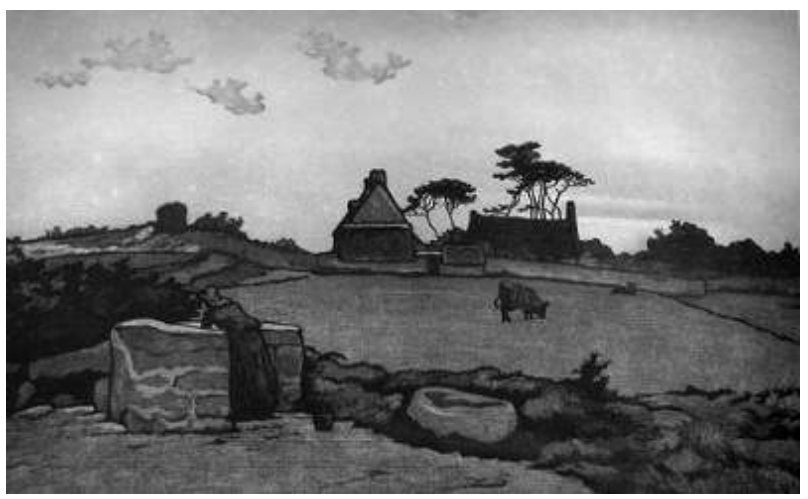

Figure 2 : Le soir sur l'île de Bréhat (aquatinte, $\mathrm{XIX}^{\mathrm{e}}-\mathrm{XX}^{\mathrm{e}}$ siècle). Source : H.-G. Meunier

Evening on Brehat Island (aquatint, $19^{\text {th }}-20^{\text {th }}$ century). Source: H.-G. Meunier

historique bréhatin ${ }^{2}$, par les archives départementales $^{3}$, et via des travaux universitaires (Brigand, 2002; Salomé, 2006) et rapports (Bedrani et Roche, 2017; Viart, 2019).

Aujourd'hui, les blogs et réseaux sociaux alimentés par des touristes, des résidents secondaires et des locaux se font le relais de ces images, y mêlant parfois une iconographie originale, témoignant de situations pittoresques davantage qu'elle ne renseigne sur les transformations de l'activité agricole.

Plus récemment, à la demande d'une association locale favorable à l'essor de l'agriculture sur l'île, un diagnostic agricole a été réalisé (Bedrani et Roche, 2017). Ce travail à la fois descriptif et prospectif, qui a vocation à dynamiser le débat politique sur la place de l'agriculture à Bréhat, est annexé au Plan local d'urbanisme (Viart, 2019). Il constitue dans ce paysage la première synthèse sur l'activité agricole publiée depuis celle de G. Vergez-Tricom.

\section{Matériaux et méthodes de l'enquête ethnographique}

Dans le cadre du projet de recherche Paroles et chemins de l'agriculture littorale, qui a pour enjeu de documenter les spécificités et les transformations de l'agriculture sur les littoraux bretons de 1945 à nos jours, une enquête ethnographique a été réalisée à Bréhat d'octobre 2017 à février 2020 (Cardinal,

2. Voir notamment le Musée virtuel de l'île de Bréhat : [https://musee-virtuel-brehat.fr/], le site de Locoven : [http://locoven.over-blog.com/] et celui de Marikavel : [http://www.marikavel.org/].

3. Les archives départementales des Côtes d'Armor [http://sallevirtuelle. cotesdarmor.fr/]
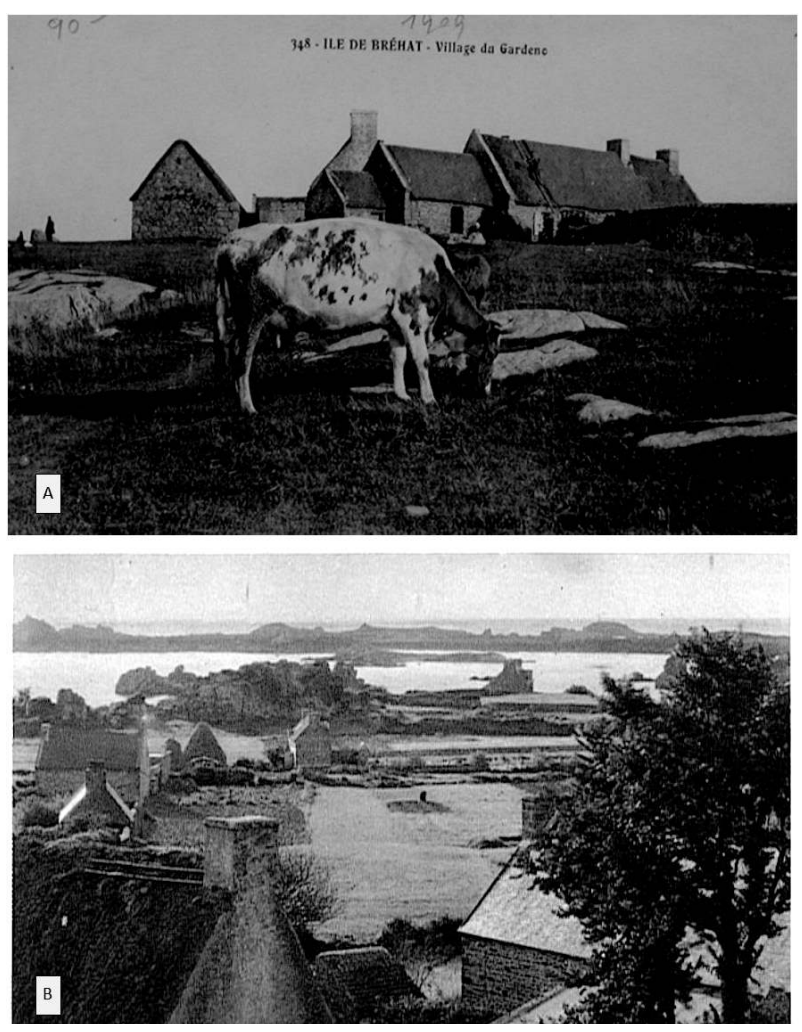

Figure 3 : Cartes postales du $1^{\text {er }}$ quart du $\mathrm{xx}^{\mathrm{e}}$ : (A) Habitat traditionnel agricole au Gardenno (collection particulière). (B) Vue partielle de l'île de Bréhat. Sources : (A) Archives départementales des Côtes d'Armor; (B) Éditions G.-L. Arlaud, 1931. (C) Collection privée J.-C. Even

Postcards, $1^{\text {st }}$ quarter of the $20^{\text {th }}$ century: (A) Traditional housing in the Gardenno district (private collection). (B) Partial view of Brehat Island. Sources: (A) Côtes d'Armor Departmental Archives; (B) G.-L. Arlaud publishings, 1931. (C) J.-C. Even private collection

2018). Bréhat est le seul territoire insulaire concerné par l'enquête. Dans la perspective retenue par ce programme, l'insularité est considérée comme une forme particulière de littoralité, caractérisée par une rupture géographique avec le continent, une intensité de l'emprise socio-spatiale d'activités économiques concurrentes, et une forte attractivité.

Le corpus de données collecté est constitué de 16 entretiens avec des agriculteurs et agricultrices, élus, membres d'associations, résidents et résidentes permanents et secondaires, d'observations directes à l'occasion de trois réunions publiques, ainsi que d'une immersion sur l'île entre janvier 2019 et février 2020. Il est complété par un travail photographique, et par la collecte de coupures de presse et de données publiées sur Internet par des résidents de l'île et des touristes. Nous avons adopté pour l'analyse une démarche régressive (Barthe et al., 2013), dans laquelle: 
«L'enquête se donne [...] pour objet non seulement d'expliquer les contraintes qui pèsent sur les situations du présent ou, indissociablement, les ressources qui y sont mises à disposition des acteurs, mais encore de permettre d'observer différemment de telles situations, en s'interrogeant sur les raisons pour lesquelles certains héritages du passé n'y sont pas actuellement activés » (Barthe et al., 2013 : 183).

La démarche d'historicisation effectuée ici articule de ce fait les données hétérogènes recueillies lors de l'enquête ethnographique avec les diverses sources historiques évoquées plus haut. Ces éléments constituent autant d'indices de la concrétisation de pactes territoriaux dans les réalités locales et quotidiennes de Bréhat, passées et présentes.

\section{QUAND LES TERRES CHANGENT DE VALEUR : UNE SOCIÉTÉ PAYSANNE QUI S'OUVRE AU TOURISME (XVIII ${ }^{E}$ SIĖCLE-ANNÉES I950)}

\section{La société paysanne à Bréhat}

À l'instar de la plupart des îles du Ponant, l'économie bréhatine se maritimise au XVIII ${ }^{\mathrm{e}}$ siècle (Guillemet, 2000). Cela conduit à une répartition genrée des activités : "Les hommes sont presque tous marins, au service de l'État ou embarqués sur les longs courriers » (Rigaud, 1890). Cette économie mixte terre-mer s'accompagne d'une organisation sociale peu hiérarchisée jusqu'aux années 1950, caractérisée par un mode de vie paysan, régulé par les cycles agraires, les périodes de coupe du goémon et les périodes de pêche (Vergez-Tricom, 1925). Chaque foyer dispose de terres - en propre et à usage collectif -, et pratique la polyculture-élevage. La féminisation de l'agriculture, la faible intégration de l'île dans des circuits commerciaux ${ }^{4}$ et le caractère égalitaire de l'organisation sociale conduisent à une parcellisation extrême des propriétés (les surfaces oscillant entre 100 et $500 \mathrm{~m}^{2}$ ), permettant ainsi de répartir une ressource territoriale et donc de subsistance - limitée (Vergez-Tricom, 1925; Le Pache et Le Pache, 1991; Menguy, 2005; Guillemet, 2000 : 73).

4. Seule l'exportation de homards vers l'Angleterre au début du XVII siècle marque l'intégration de l'île dans des circuits commerciaux d'importance (Guillemet, 2000).
En partant des estimations des surfaces allouées aux divers usages du territoire (figure 4), il est difficile d'appréhender l'évolution de l'usage des terres entre le $\mathrm{XIX}^{\mathrm{e}}$ et le début du XX $\mathrm{XX}^{\mathrm{e}}$ siècle. Ces données mettent malgré tout en évidence l'emprise spatiale des activités agricoles, qui occupent entre les deux tiers et la quasi-totalité des 305 ha de l'île.

L'ensemble des témoignages, écrits comme iconographiques, atteste que l'île est peu arborée et rasée par les vents et le pâturage (Guillemet, 2000). Les pommes de terre primeurs ${ }^{5}$ sont exportées à partir de 1890 et constituent dès lors une grande richesse pour les habitants de l'île qui se traduit par le fait que chaque famille dispose également d'un cheptel conséquent, " au moins 1 ou 2 vaches, quelques porcs et 3 ou 4 moutons » (Vergez-Tricom, 1925).

«Autrefois, l'île de Bréhat était renommée pour les primeurs. [...] Il y avait la vedette de marchandises, je crois qu'elle partait jusqu'à Saint Malo les livrer. Et après, ça partait sur les ferries, en Angleterre... C'était avant le marché commun, ça. Rien que de vendre 3 sacs, c'était un apport d'argent. C'était un marché très, très précieux » (habitante de Bréhat, 2019).

Cette activité lucrative prend fin en effet à l'instauration du marché commun, en 1957.

\section{La déstabilisation de l'économie vivrière}

À la toute fin du XIX ${ }^{\mathrm{e}}$ siècle, les règles et coutumes d'usage des terres évoluent. Tandis que la règle de la vaine pâture ${ }^{6}$ était " autorisée par un usage immémorial ${ }^{7}{ }$ dans les terres non encloses, la multiplication des conflits entraîne des modifications dans l'organisation socio-spatiale de l'agriculture. La loi de l'égalité d'accès et d'usufruit aux parcelles agricoles non ensemencées, qui prévalait, tombe en déshérence (Vergez-Tricom, 1925; Le Pache et Le Pache, 1991; Guillemet, 2000). L'affirmation des droits de propriété se matérialise notamment par la clôture des parcelles via des murets de pierres sèches ou des choux fourragers

5. Pommes de terre plantées et récoltées précocement, habituellement commercialisées à partir de Pâques.

6. Droit d'usage qui permet de faire paître gratuitement son bétail sur des terres communes ou non clôturées et les bords de chemin, ici les vaches et les moutons.

7. Voir arrêt de la Cour de cassation de 1867 (France, $1867: 443$ ). 


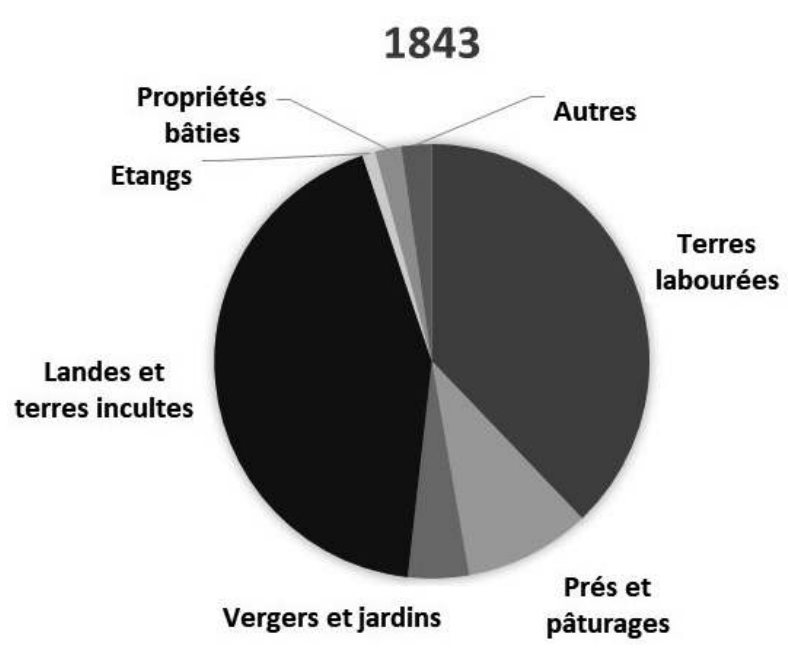

1925

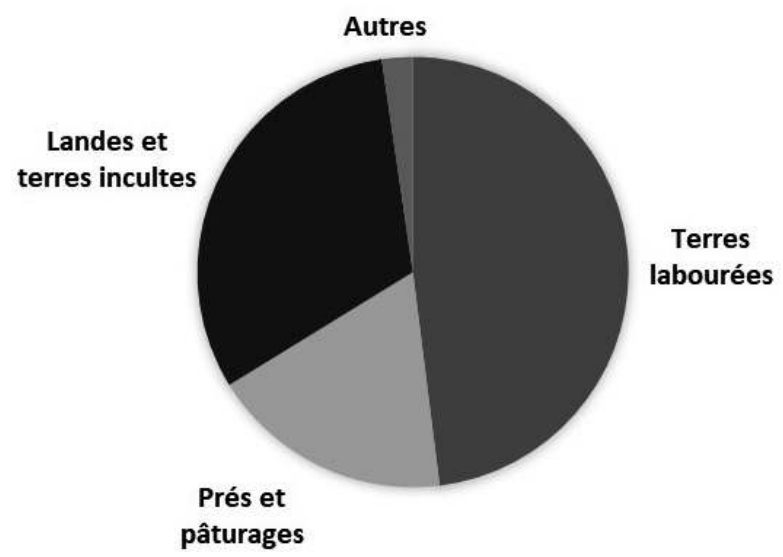

Figure 4 : Répartition des divers usages du foncier à Bréhat en 1843 (d'après les catégories et les chiffres de Marteville et Varin, 1843 ) et en 1925 (d'après les catégories et l'estimation des surfaces correspondantes par Vergez-Tricom, 1925). Source : S. Dupé Land-use repartition in Brehat in 1843 (according to Marteville and Varin's categories and figures, 1843) and in 1925 (according to Vergez-Tricom's categories and the estimate of the corresponding surfaces, 1925). Source: S. Dupé

(figure 5). Le caractère égalitaire de l'organisation sociale locale est affaibli, laissant émerger l'individualisme agraire (Laligant, 2008). Au tournant du $\mathrm{Xx}^{\mathrm{e}}$ siècle, la pêche lointaine prend fin pour les bréhatins et la pêche locale pâtit de la concurrence avec les pêcheurs continentaux. L'économie maritime s'effondre (Vergez-Tricom, 1925). Cette transformation bouleverse l'organisation socio-économique de l'île à deux titres : les hommes investissent les activités agricoles et le port est réaffecté à d'autres usages, comme le transport de voyageurs. L'influence croissante du tourisme constituera désormais le moteur central de la déstructura- tion de la société paysanne bréhatine. En 1907, la première vedette reliant quotidiennement l'île au continent est mise en circulation, accélérant l'arrivée de voyageurs qui viennent s'établir quelques semaines ou mois :

«En 1817, le maire déclare qu'il ne venait pas, par an, 20 voyageurs dans l'île, c'est aujourd'hui par centaines qu'on les compte. Aujourd'hui, la population de l'île double presque en été : les hôtels se sont construits, et aux alentours du Bourg, les maisonnettes basses ont été remplacées par de pimpantes villas couvertes d'ardoises et louées pour la saison ». (Vergez-Tricom, 1925 : 53)

Nombreuses sont les sources qui attestent de l'attrait précoce de Bréhat pour les artistes et intellectuels qui fréquentent l'île (idem; Huchet du Guermeur, 2000; Brigand, 2002; Buhot, 2005 , Salomé, 2006). Ce sont à la fois les paysages littoraux, l'isolement de l'île, et l'entre-soi, qui attirent les élites intellectuelles parisiennes et bretonnes (Corbin, 1990; Huchet du Guermeur, 2000; Buhot, 2005). Au moment de l'essor des stations balnéaires bretonnes, Bréhat s'inscrit durablement dans un tourisme élitaire et résidentiel (Huchet du Guermeur, 2000). L'inscription de l'île comme premier site classé de France en 1907 témoigne de l'influence et de l'intérêt de ces premiers voyageurs pour un territoire dont ils veulent préserver les paysages pittoresques. Cet attrait touristique va pourtant profondément modifier l'organisation socio-spatiale de l'île et ses paysages.

Avec l'arrivée des congés payés, les résidents secondaires recrutent des femmes bréhatines pour entretenir la maison et garder les enfants, et des hommes pour entretenir les jardins (com. pers, habitante de Bréhat, 2019). Peu à peu, l'économie bréhatine se tourne vers les " parisiens qui villégiaturent »(Vergez-Tricom : 47), initiant une économie de service orientée vers le tourisme, qui concerne 75,5\% de l'activité bréhatine en 2015 (Insee, 2015).

\section{L'attraction du littoral, une ressource qui s'insère dans l'économie vivrière}

À Bréhat, les régulations politiques nationales et régionales sont faibles, l'île bénéficiant de régimes d'exception du fait de son emplacement stratégique et de son isolement (Le Pache et Le Pache, 1991, 


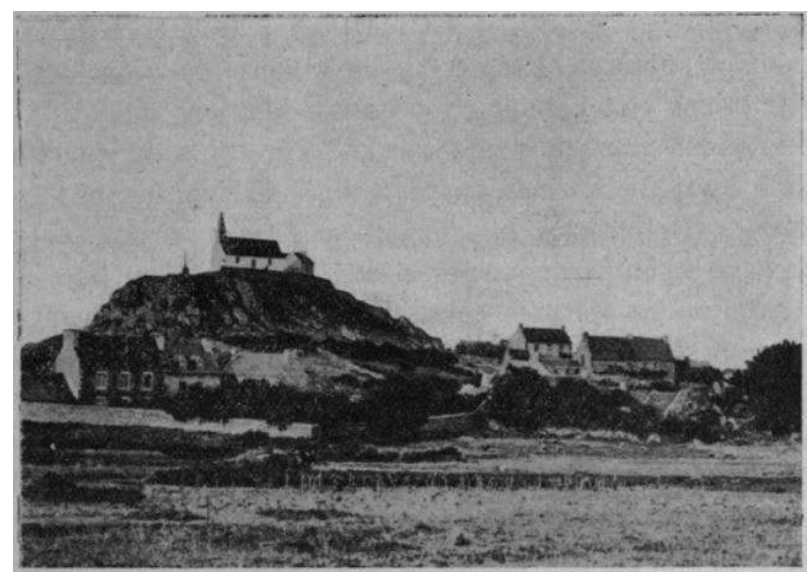

Figure 5 : Le mont Saint-Michel à l'île de Bréhat et champs entourés de choux fourragers. Source : Vergez-Tricom, $1925: 49$

The Mont Saint-Michel in Brehat and fields surrounded by collards. Source: Vergez-Tricom, $1925: 49$

Guillemet, 2000). Ce qui déstabilise une organisation sociale globalement égalitaire et homogène, dans laquelle l'agriculture et la pêche structurent l'espace social local depuis le XVIII ${ }^{\mathrm{e}}$ siècle, c'est une conjonction de facteurs exogènes, partiellement réappropriés localement : les transformations sociotechniques dans le domaine du transport maritime, la fin de l'exportation des pommes de terre et l'arrivée de capitaux extérieurs via le tourisme. Cela se traduit, au début du $\mathrm{Xx}^{\mathrm{e}}$ siècle, par une modification de la valeur symbolique des terres

« Ces petits propriétaires d'un ou deux hectares en moyenne, de huit au plus, se disputent âprement, surtout depuis la guerre, les parcelles de terre dont les prix, aux jours de vente, montent à des dix mille francs l'hectare » (Vergez-Tricom, 1925 : 56).

La conjonction de l'individualisme agraire et de l'attraction mondaine pour l'insularité - avec la diversification de ressources qu'elle offre -, conduit à une réallocation des terres, inédite par son ampleur.

Les sources disponibles ne permettent pas d'apprécier finement le poids respectif de ces facteurs de déstabilisation, ni de déterminer à quel moment les changements qui s'opèrent deviennent déterminants; la nature et la vitesse de la rupture entre un mode de vie basé sur une économie vivrière et une économie de service tournée vers le tourisme ne sont pas documentés.

\section{LITTORALISATION ET DÉSTRUCTURATION D'UN MONDE RURAL (I960-20I0)}

\section{De l'usage vivrier à l'usage récréatif du territoire}

La seconde partie du $\mathrm{Xx}^{\mathrm{e}}$ siècle correspond à un effondrement de la place de l'activité agricole sur l'île. Le caractère insulaire de Bréhat, son éloignement des nouvelles voies de transport et la mécanisation « impossible sur l'île » (Menguy 2005 : 335) la maintiennent de fait à l'écart du grand mouvement de modernisation agricole des années 1960 ; cette rupture est restée dans les mémoires. Le nombre d'agriculteurs chute drastiquement. En 1955, 25 exploitations sont recensées (Bedrani et Roche, 2017). Une ancienne agricultrice rapporte que dans les années 1960, il y a 5 ou 6 fermes sur l'île Nord, contre 2 sur l'île $\mathrm{Sud}^{8}$. Bréhat apparaît ainsi, par comparaison avec d'autres îles du Ponant, comme une île à « l'activité agricole limitée » (GarigliettiBrachetto, 2013). Les cultures, landes et pâtures, qui couvraient l'essentiel du territoire jusqu'en 1925, ne recouvrent plus qu'un tiers de la superficie de l'île en 1977 (figure 6), puis le 10 e seulement en 2017 (Bedrani et Roche, 2017). Les causes de cette spectaculaire marginalisation sont multiples.

Le principal facteur est la mise en tourisme de l'île. L'augmentation de l'emprise spatiale du tourisme élitaire et résidentiel s'accompagne d'un fort déclin démographique (figure 7). À partir des années 1950, l'accélération du tourisme intensifie les ventes de terrain et les conflits qui y sont associés (Huchet du Guermeur, 2000). Et dès 1950, la part de résidences secondaires dépasse celle des résidences principales (figure 8). Cette bascule bouleverse la structure économique et sociale de l'île. L'habitat des résidents secondaires est concentré sur la zone côtière, tandis que l'habitat permanent est dispersé dans les zones les moins attractives (Buhot, 2005). L'attraction paysagère du littoral a amplement conditionné la réorganisation socio-spatiale de l'île vers un usage récréatif saisonnier.

Dans le sillage de la résidentialisation, le développement du tourisme journalier s'est amplifié à partir des

8. Il y en a au moins une troisième, qu'elle ne comptabilise pas, à laquelle s'ajoute la ferme basée sur Béniguet. Ce chiffre évoqué par l'enquêté est corroboré par les recensements agricoles des décennies qui suivent. 


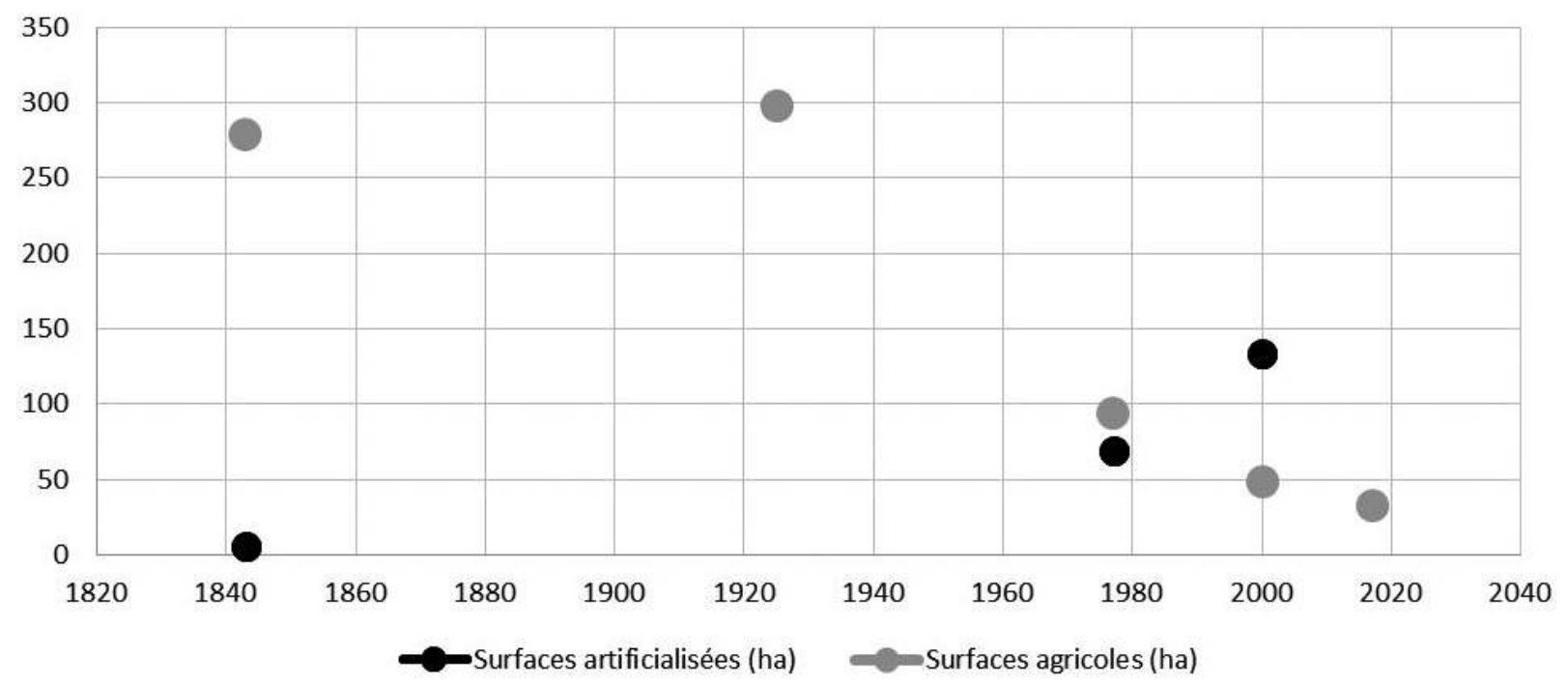

Figure 6 : Chute drastique de la surface de terres agricoles : en 1977, il ne reste plus qu'un tiers de celles utilisées en 1925. Source : S. Dupé, d'après Marteville et Varin (1843), Vergez-Tricom (1925), Bedrani et Roche (2017) et Viart (2019)

Drastic drop in the area of farming land: in 1977, only a third of those used in 1925 remain. Source: S. Dupé, based on Marteville and Varin (1843), Vergez-Tricom (1925), Bedrani and Roche (2017) and Viart (2019)

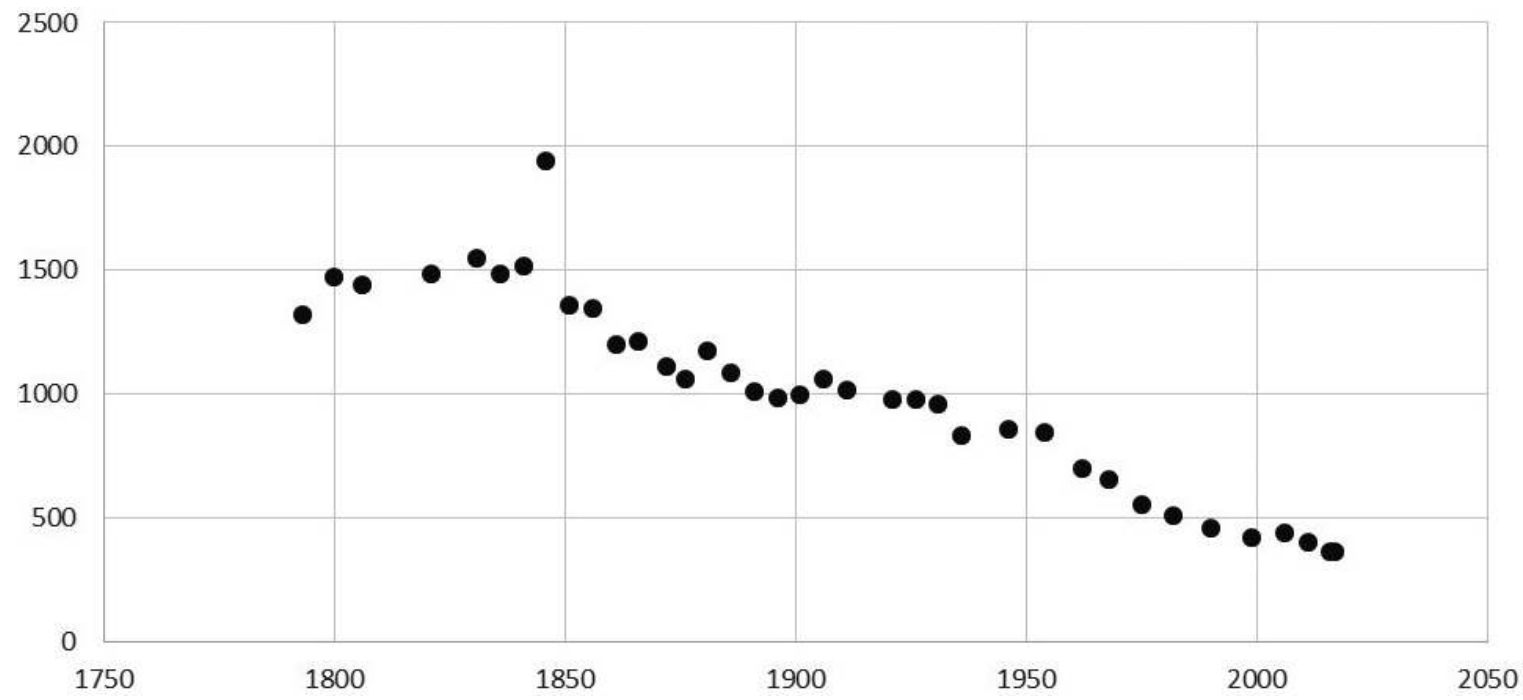

Figure 7. Évolution démographique de Bréhat de 1793 à 2017. Source : S. Dupé, d'après Index EHESS [http://cassini.ehess.fr/cassini/fr/ html/fiche.php?select_resultat=17489\#] jusqu'en 1999 puis Insee à partir de 2006

Demographic trend in Brehat from 1793 to 2017. Source: S. Dupé, based on the EHESS Index until 1999, Insee from 2006

années 1980. Si en 1980, on compte 225000 passagers, ils sont 400000 en 2003 (Buhot, 2005 : 51).

La profonde réorganisation des usages du territoire vers un tourisme résidentiel et journalier a produit successivement deux effets pour l'activité agricole : d'abord sa régression, puis sa stabilisation (figure 6). La dynamique d'artificialisation des sols est cependant freinée par l'élaboration d'un premier plan d'occupation des sols en 1973, puis par un empilement de dispositifs de protection des sites ${ }^{9}$ (idem). L'effectivité de ces outils de régulation foncière demeure relative jusqu'en 2000. Cette politique tardive traduit sa faible importance politique locale ${ }^{10}$ et ne permet que de maintenir un tissu agricole déjà très déstructuré.

9. Site classé, Loi littoral, sites inscrits, Zones naturelles d’intérêt écologique, faunistique et floristique, convention Ospar, Zone spéciale de protection, site d'intérêt communautaire.

10. Une enquêtée affirme qu' « il n'y a pas eu l'affirmation d'un projet » (résidente secondaire, 2019). 


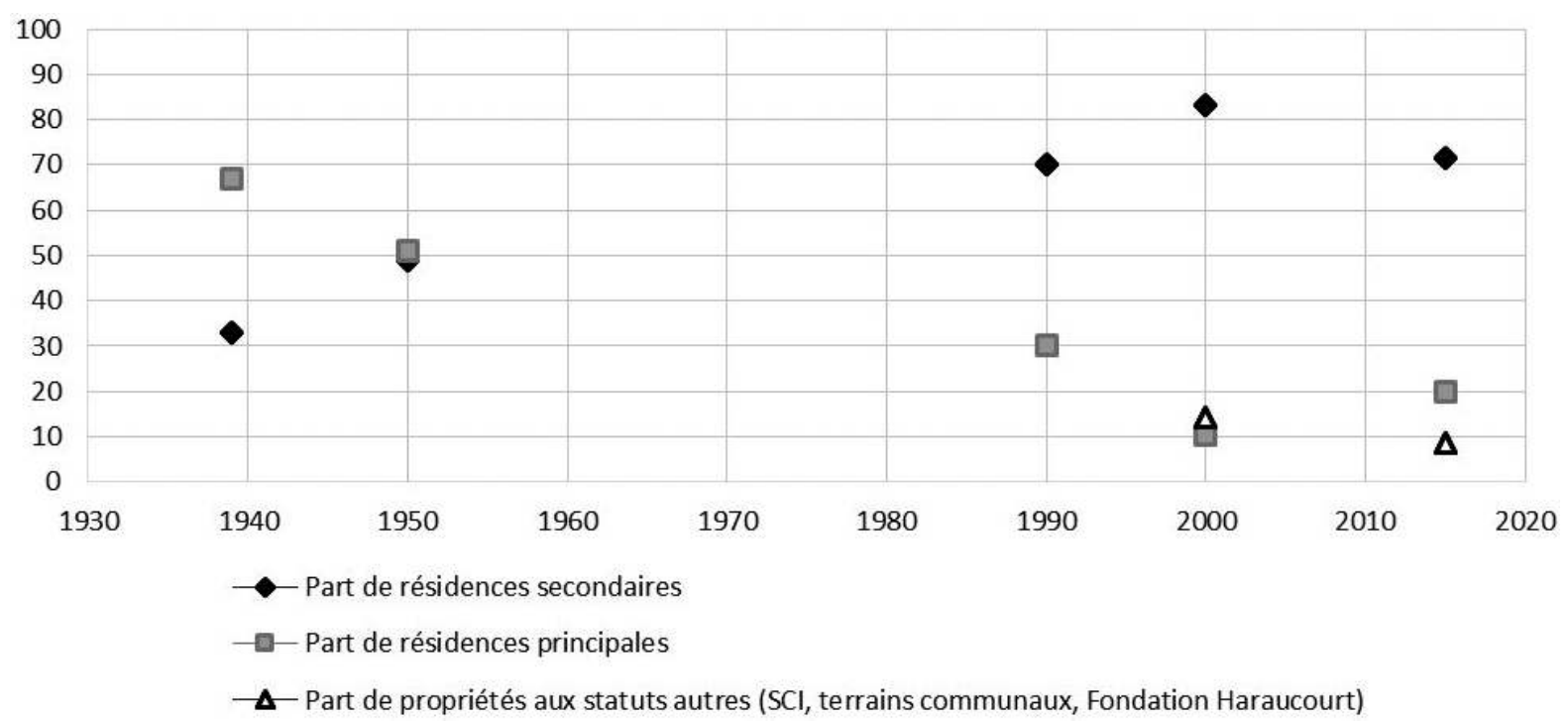

Figure 8 : Evolution de la répartition de la propriété résidentielle à Bréhat de 1939 à 2015. Source : S. Dupé, d'après Buhot (2003), Buhot (2005) Insee (2015)

Evolution of residential property in Brehat from 1939 to 2015. Source: S. Dupé, based on Buhot (2003), Buhot (2005), Insee (2015)

\section{Marginalisation et subsistances de l'agriculture}

En même temps que l'agriculture perd de l'importance politique, spatiale, économique et symbolique, elle n'est plus valorisée dans les trajectoires individuelles. Au-delà de son impact spatial, la mise en tourisme de lî̀le a aussi eu des effets sur les choix de vie des agriculteurs et sur leur type de production.

Le développement d'une économie de service à Bréhat modifie les stratégies professionnelles au-delà des schémas de transmission qui pouvaient prévaloir antérieurement. Les enfants d'agriculteurs peuvent " sortir » de l'île, saisissant la dynamique de massification scolaire des années 1960 qui ouvre des horizons professionnels. Mais ils optent aussi souvent pour une insertion dans la nouvelle économie de service insulaire : « les personnes n'allaient plus travailler les champs, parce qu'elles étaient employées chez les touristes » (habitante de l'île, 2019).

Plusieurs agriculteurs deviennent polyactifs, assurant la pérennité d'une activité agricole en réalisant parallèlement des activités plus rémunératrices : bâtiment, hôtellerie, restauration, entretien des propriétés, transport de charbon, évacuation des ordures, enrobage des routes, etc. Les diversifications peuvent se penser comme des extensions, voire comme une voie de sortie des activités agricoles, qui s'appuient sur la possession de matériel professionnel adapté.
La modernisation agricole n'a trouvé qu'une faible résonance dans l'agriculture bréhatine. Bien que René Dumont ${ }^{11}$ ait recommandé dès 1948 lors de sa visite à Bréhat de "se hâter de remembrer, et au moins ne pas entraver $[\ldots]$ la concentration de la culture en quelques fermes assez importantes » (Dumont, 1951), les limites spatiales intrinsèques à l'espace insulaire complexifient le développement de ce modèle productiviste, basé sur l'affranchissement des contraintes environnementales (Barbier et Goulet, 2013) et l'insertion d'exploitants dans des circuits dits conventionnels. Tandis que dans les années 1950-1960, la Bretagne se spécialisait et intensifiait sa production (animale et légumière) par la mécanisation et se tournait vers l'exportation (Canévet, 1992), une seule ferme s'est insérée dans ce modèle, dans les années 1960. Avec une quarantaine de vaches laitières, elle concentrait une grande partie des terres agricoles de lîle Sud, et était dotée d'une salle de traite, « la quatrième des Côtes d'Armor, la plus moderne à l'époque! » (habitante, 2018). L'exploitation n'a toutefois pas été reprise. D’autres tentatives d'insertion des agriculteurs locaux dans ce modèle régional basé sur la déterritorialisation (Rieutort, 2009) ont existé, comme l'exportation de légumes et de viande via des coopératives situées

11. René Dumont était alors conseiller agricole au Commissariat Général du Plan de modernisation et d'équipement. 
sur le continent. Mais les contraintes insulaires ont découragé les agriculteurs :

« La coop, là, j’ai arrêté. C'était le boxon, des fois ils n'avaient pas de camion de ramassage, je me retrouvais avec des bêtes sur le continent. Un jour je me suis retrouvé : "Ah ben non on verra ça demain!" Mais j'habite sur une île là! » (agriculteur de l'île, avril 2018).

Les possibilités d'un redéploiement agricole rémunérateur à Bréhat résident alors dans le développement de productions à haute valeur ajoutée (Dumont, 1951; Péron, 1993; Menguy, 2005). Quelques initiatives de diversification de production émergent, comme une production de fleurs dans les années 1960 « jusqu’à l'arrivée des Hollandais sur le marché français » (habitante de Bréhat, 2018), l'élevage de taurillons, etc. Cela ne suffit pas à enrayer un décrochage massif. Les agriculteurs doivent en effet faire face à la raréfaction du foncier, aux contraintes logistiques associées à l'insularité, ainsi qu'à l'inadaptation au cadre réglementaire national et européen : l'abattoir de l'île ferme en 1972, ne respectant plus les nouvelles règles sanitaires (Menguy, 2005), et les déclarations PAC sont complexifiées par le morcellement du foncier.

Entre les années 1980 et 2000, quelques agriculteurs s'installent hors cadre familial. Ces néo-insulaires (Huchet du Guermeur, 2000), n’héritent pas de la condition insulaire mais choisissent de l'adopter (Péron, 1993). Ils sont qualifiés ainsi par analogie avec les trajectoires des néo-ruraux avec qui ils partagent une volonté d'implication dans la vie locale et l'insertion dans des modèles agricoles alternatifs (Hervieu et Hervieu-Léger, 1979). Si leur installation est facilitée par un coût du foncier encore relativement bas, l'accès à la terre est complexifié par la multiplicité de propriétaires par ailleurs rarement présents (Buhot, 2005) et la difficulté à gagner la confiance des Bréhatins dits «de souche ${ }^{12}$ » pour accéder à la location des terres.

\section{L'abandon de la ruralité}

L'agriculture s'efface des mémoires locales au cours du second pacte territorial, caractérisé par la marginalisation de l'agriculture. Les éléments issus d'entretiens sont flous ou contradictoires, voire inexistants. Ce silence relatif est, en lui-même, significatif de cette perte d'importance. Les agriculteurs ne parviennent pas, à cette époque, à se politiser pour défendre et mettre en débat la place de l'agriculture sur l'île. Dans le débat public, l'agriculture apparaît davantage comme source de nuisances diverses, perturbant un lieu de villégiature exceptionnel. La démission du Conseil municipal en 1970 s'insurgeant contre « la mise en circulation d'un huitième tracteur agricole dont les pétarades [...] troubleraient la quiétude de la population estivale, attirée précisément par le charme, et surtout par le calme de l'archipel » (cité par Menguy, 2005 : 302) témoigne de la marginalisation symbolique de l'activité agricole sur le territoire. De plus, elle ne s'intègre pas dans les dynamiques d'esthétisation et de patrimonialisation précocement apparues sur l'île. Elle incarne le banal et rompt avec l'insularité mythique qui fait de l'île l'« envers du monde continental » (Péron, 2005 : 782). Les conflits d'usage se multiplient :

« Il y a la concurrence [foncière] qui est liée vraiment au désir des résidents d'avoir la paix, quoi [rire]! De pas courir après les vaches ou les taureaux, a fortiori. [...] Tout le monde était complètement affolé à l'idée que les petits taureaux s'échappent » (résidente secondaire, 2019).

La déprise agricole puis la déstructuration du monde rural qui marquent la seconde moitié du $\mathrm{Xx}^{\mathrm{e}}$ siècle en France (Rieutort, 2009; Hervieu et Purseigle, 2013) sont ainsi plus radicales à Bréhat. L'insularité contribue à ce décrochage massif dès la fin des années 1950, la résidentialisation précoce de l'île constituant un facteur supplémentaire de déstabilisation.

La disjonction entre les dimensions travail, œuvre et action de l'activité agricole caractérise cette marginalisation spatiale, sociale et symbolique de l'agriculture. À Bréhat, la fin des paysans (Mendras, 1967) est ainsi aussi une fin de la ruralité, dans la mesure où disparaît, au cours de la période 19502010, l'espace de proximité dans lequel se déployait une sociabilité spécifique, fondée sur l'intensité des interactions entre production alimentaire, gestion de l'espace et consommation.

12. Pour reprendre une terminologie fréquemment employée par les enquêtés. 


\section{L'ANCRAGE DE L'AGRICULTURE DANS UNE RURALITÉ RÉINVENTÉE (DEPUIS 20IO)}

\section{Densification de l'activité agricole sur un foncier contraint}

Le troisième pacte territorial est marqué par une réapparition de l'agriculture dans l'espace social local. Il s'instaure dans un contexte où les exploitations agricoles demeurent rares et sont diversifiées : élevages laitiers et viande, pépinière, maraîchage. Le nombre d'exploitants installés reste stable (4-5), mais trois exploitantes se sont installées depuis 2012, tandis que le nombre de salariés agricoles augmente régulièrement. En parallèle, une nouvelle catégorie d'acteurs agricoles émerge : des personnes au statut hybride - particuliers, auto-entrepreneurs, entrepreneurs non agricoles, agriculteurs installés sur le continent et présents à la saison touristique - qui produisent, transforment et vendent leurs produits agricoles à Bréhat (figure 9). Quelques projets d'installation agricole voient le jour, sans se concrétiser (ils ne sont pas figurés sur ce schéma). Pour asseoir cette dynamique, le principal facteur limitant est la disponibilité des terres et bâtiments agricoles, mais également de logements :
« Je me détourne de l'agricole à la longue... On n'aboutit à rien, on n'a pas de bâtiments corrects... T'as pas de bail de 15, 20 ans, 40 ans pour être peinard...» (agriculteur, 2018).

Les surfaces agricoles utiles ont en effet peu de chances de s'accroître; la concentration d'activités agricoles sur une très petite surface (32ha) (figure 10) incite ainsi les producteurs et transformateurs à déployer un ensemble de stratégies d'adaptation selon trois axes : l'adaptation des pratiques culturales à un foncier restreint, l'inscription de l'activité des exploitations dans l'économie touristique et l'instauration de nouvelles alliances favorisant l'accès aux terres.

Dans la conduite des exploitations, l'adaptation à un faible accès au foncier se manifeste par une intensification de la production, qui permet de produire plus de valeur sur une surface limitée. Selon les exploitants, l'intensification passe soit par une hausse significative de la masse salariale à la saison touristique (figure 9), soit par des pratiques culturales qui tirent parti au mieux des ressources (filets de forçage, production sous serre, rotations courtes), soit enfin par l'apport de fourrages - en partie importés - aux bovins sur la zone de pâturage (figure 11).

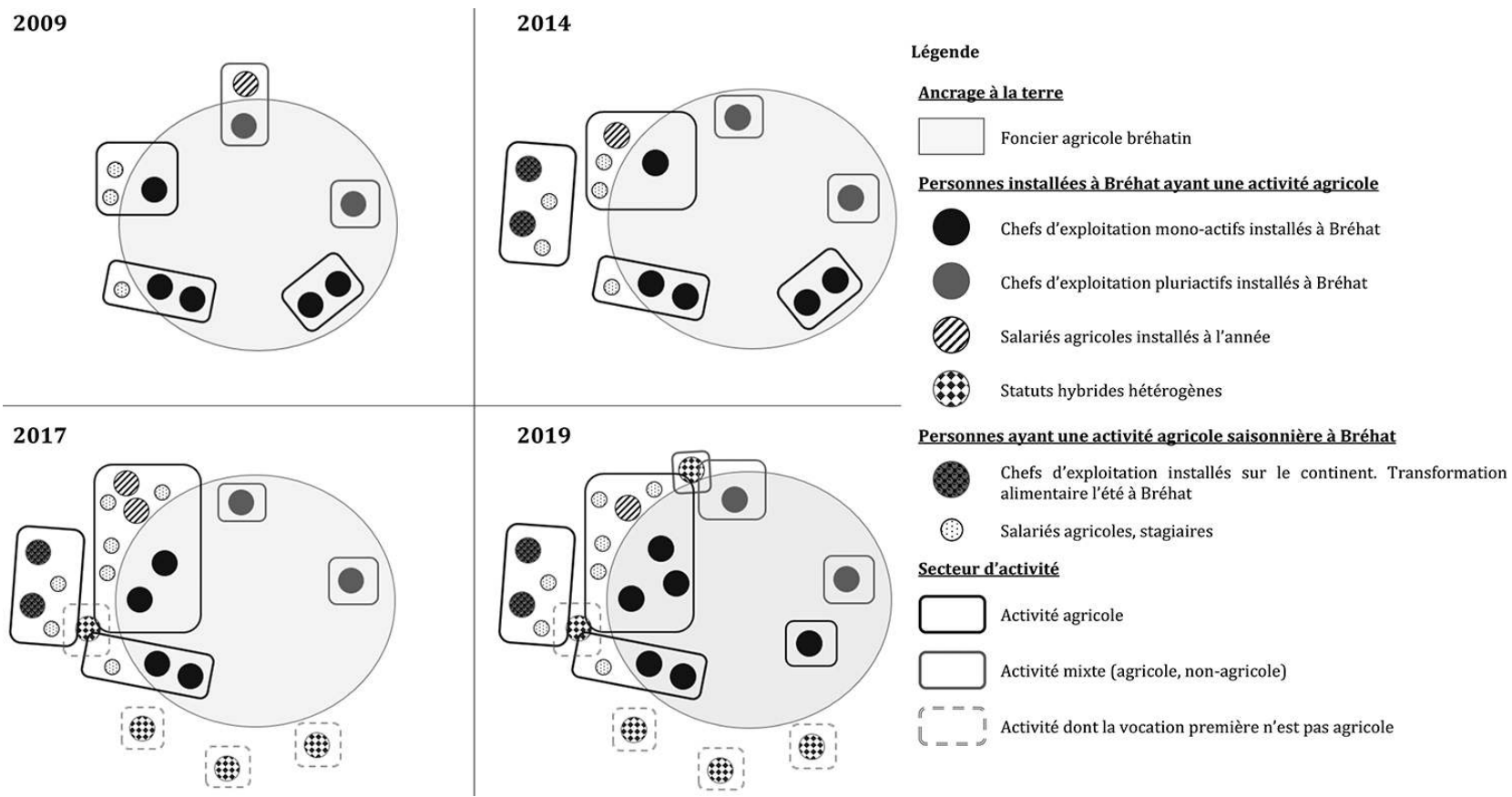

Figure 9 : Activité agricole à Bréhat en 2009, 2014, 2017 et 2019. Source : S. Dupé, d'après les données recueillies lors de l'enquête ethnographique

Agricultural activity in Brehat in 2009, 2014, 2017 and 2019. Source: S. Dupé, from data collected during the ethnographic survey 


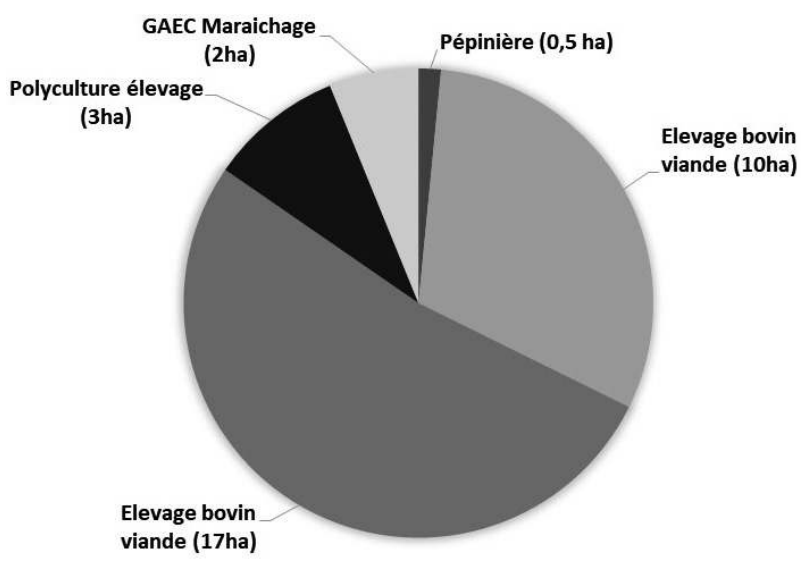

Figure 10 : Répartition des surfaces agricoles par exploitation en 2017, d'après Bedrani et Roche (2017) and Roche (2017)

Farmland repartition per farm in 2017, based on Bedrani

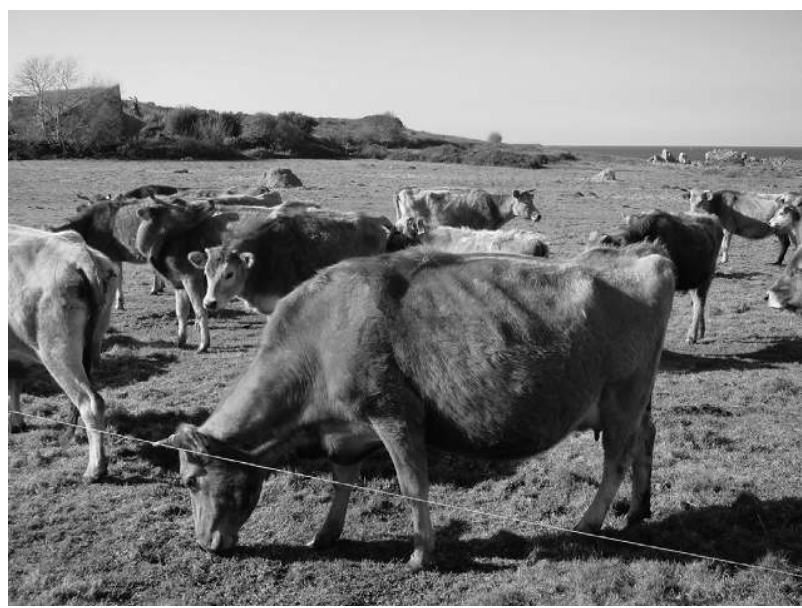

Figure 11 : Bovins « à viande » sur une prairie alimentée en fourrage. Crédit photo : S. Dupé

Cows from a beef farm fed by grazing and fodder on a grassland. Photo credits: S. Dupé

Le difficile accès à la terre fait aussi émerger une stratégie de production sans terres. Les maraîchers valorisent par exemple des ressources marines, collectées sur l'estran (transformation d'algues). Pour l'ensemble des producteurs qui ne sont pas installés comme exploitants, l'enjeu est de développer des modalités de production qui ne consomment pas de foncier agricole. Les boulangers importent leur farine, le transformateur de plantes sauvages collecte sur des zones interstitielles, les pépiniéristes et un auto-entrepreneur produisent des plantes ornementales en pot, et les deux apiculteurs posent leurs ruches sur des terrains privés.

\section{Dans un coin du jardin? Une activité agricole inféodée à l'économie de service}

L'économie touristique et résidentielle est une ressource qui permet de compenser la faible capacité productive des agriculteurs (Talandier, 2008). Les projets agricoles se pensent ainsi dans le cadre de «l'île aux fleurs », qui fait office de socle socioéconomique pour l'acceptation et la pérennité des projets agricoles, tout en les circonscrivant de façon rigide : " Lî̂le aux fleurs, ce n'est pas l'île aux légumes » (résident secondaire, avril 2018).

Les deux éleveurs de bovins-viande ont opté pour une diversification de leurs sources de revenus en se tournant vers une économie de service : transport, entretien de l'espace portuaire et de jardins. Ces activités « complémentaires » prennent le pas sur leur activité agricole, jusqu'à devenir une condition du maintien de leur élevage.

Quant aux autres agriculteurs, ils ont fait le choix de s'orienter vers une commercialisation locale plus rémunératrice. Ils calibrent leurs productions sur les rythmes et les modes de consommation des touristes et résidents secondaires, en recherche de produits de qualité :

«Le but, c'est hors période estivale, de pouvoir transformer mon lait pour le garder pour quand les touristes vont venir, avoir des produits, pas perdre mon lait» (avril 2018).

En combinant les plus-values sur leurs produits (labellisation, transformation, commercialisation en vente directe, élaboration de produits rares), les acteurs agricoles - disposant ou non de foncier se saisissent de la puissance symbolique « d'être ici » (Sencebe, 2004) et contribuent à entretenir l'image de marque et la mise en scène du territoire ${ }^{13}$ (figure 12). Leurs produits, sont avant tout destinés à une clientèle dotée d'un important pouvoir d'achat. Ainsi, malgré la volonté affichée par des agriculteurs de l'île de "nourrir les Bréhatins », la re-territorialisation de l'agriculture n'est économiquement viable, selon les agriculteurs, que si elle est destinée à des personnes aisées, moins présentes à l'année sur le territoire. Il y a alors dissociation entre les territoires d'origine des acheteurs et ceux de vente et de production :

13. Cela passe aussi par l'intégration de la notion paysagère dans le choix d'implantation matérielle de leurs activités sur le territoire. 
«C'est du haut de gamme hein. Un euro la pomme, euh... Donc les gens de Bréhat ils le disent, la plupart ne peuvent pas acheter ces produits » (ancien habitant de l'île, 2018).

Ce ré-ancrage de l'agriculture peut alors profiter à l'entretien d'une ruralité idéalisée, mise en scène de façon intermittente durant la période estivale. Par des stratégies d'incarnation de « l'exceptionnalité de l'île », les acteurs agricoles peuvent favoriser la légitimation d'une « ruralité empaysanée » (Mormont, 1990), contribuant à une muséification de l'île.

\section{Mise en débat de la place de l'agriculture sur le territoire}

À partir de 2017, à l'occasion de l'élaboration du Plan local d'urbanisme, la question agricole sort des sphères informelles et est mise en débat à l'échelle locale. Les premiers écrits préparatoires du projet d'aménagement communal occultent la question agricole derrière d'autres problématiques locales et entérinent l'application de lois contraignantes pour l'implantation agricole, comme la loi Littoral (Bedrani et Roche, 2017). Dans le même temps, la possibilité du maintien d'une vie à l'année à moyen
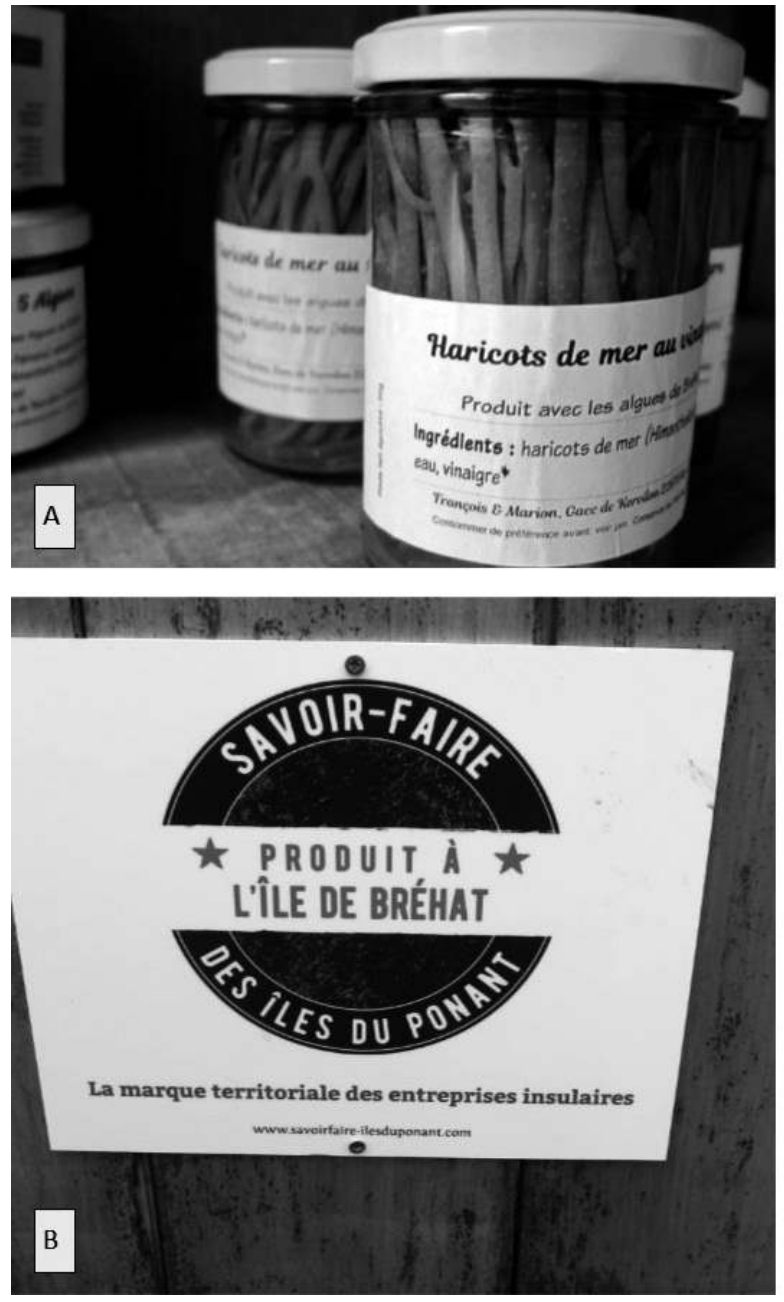

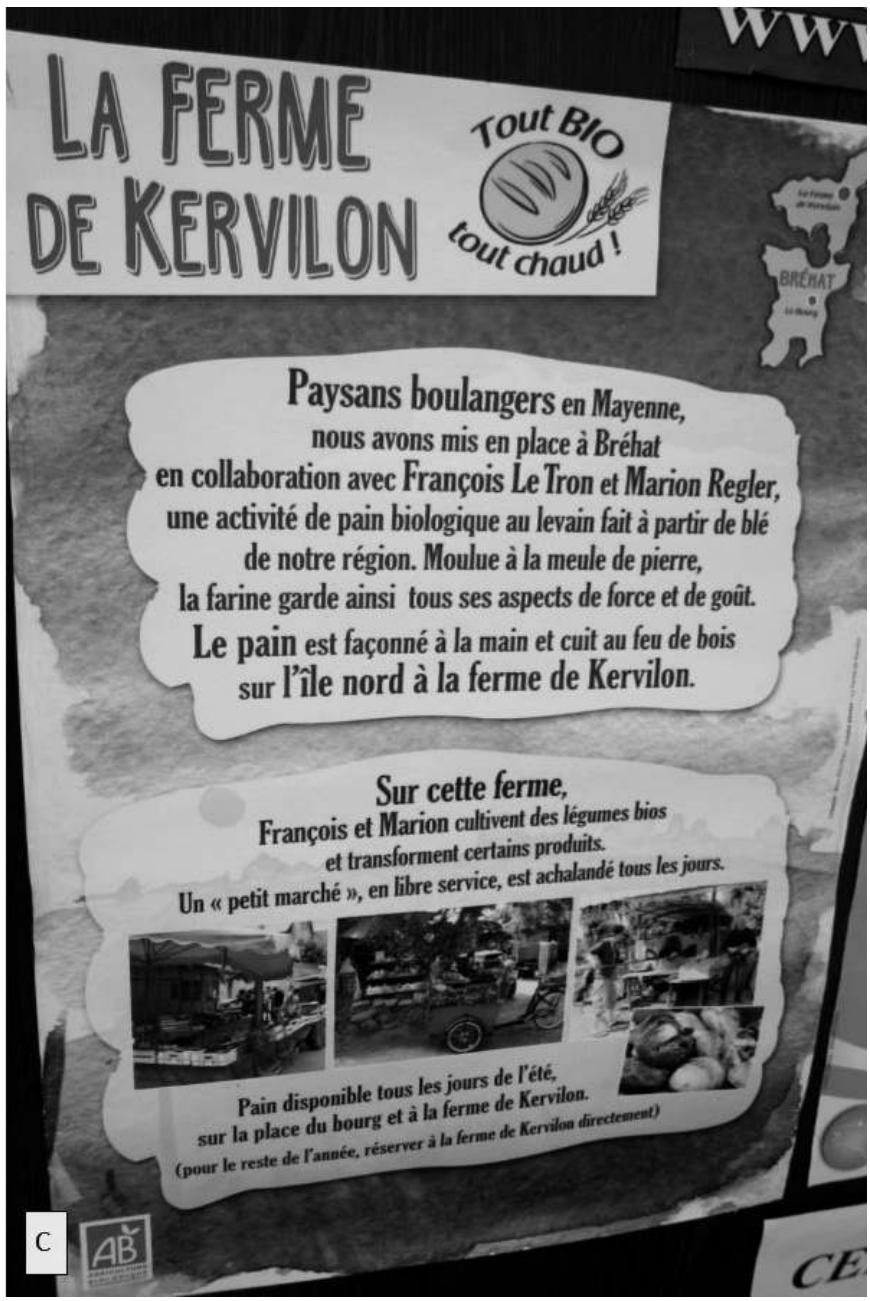

Figure 12 : Stratégies de labellisation : (A) Label "Savoir-faire des îles du Ponant ", créé par l'Association des îles du Ponant, affiché à l'entrée de la pépinière. (B) Vente d'algues transformées, labellisées Agriculture Biologique. (C) Affiche présentant les boulangers présents l'été à la ferme de Kervilon et au marché, ainsi que les maraîchers, labellisés Agriculture Biologique. Crédit photo : J. Cardinal, S. Dupé

Labellisation strategies: (A) Label «Savoir-faire des îles du Ponant ", created by the Association des îles du Ponant, showcased at the entrance of the nursery. (B) Sale of processed seaweed, with the organic farming label. (C) A poster introducing the bakers selling bread at the Kervilon farm and at the market during the summer season, with an organic farming label. Photo credits: J. Cardinal, S. Dupé 
ou long terme est questionnée. La question agricole stimule et nourrit la réflexion politique menée sur la re-légitimation de l'espace insulaire comme espace de production - et non seulement de loisirs (Péron, 2005) - et des enjeux d'accès au foncier qui y sont associés. L'insertion socio-spatiale de l'agriculture a ainsi pu trouver sa place dans l'agenda politique local.

La revendication d'une capacité de maintien voire de redéploiement - de l'activité agricole est saisie par quelques agriculteurs, par deux associations (portées essentiellement par des néo-insulaires) qui assument un rôle de contre-pouvoir vis-à-vis de la politique de la municipalité, et par quelques résidents secondaires attentifs aux problématiques agricoles. Dans ce contexte, l'élaboration du plan local d'urbanisme suscite la constitution d'un comité agricole réunissant propriétaires fonciers, agriculteurs et conseillers municipaux, configuration hybride déjà repérée en milieu périurbain (Bacconnier-Baylet, 2007). La multiplicité des ancrages socio-politiques de ses membres permet de confronter divers enjeux liés au maintien de l'activité agricole sur l'île. À l'occasion de réunions internes ou de débats publics (figure 13), le comité agricole anime des réflexions sur les types d'agriculture à développer en priorité, les instruments fonciers, les conflits autour de la dimension patrimoniale de l'île et autour des pratiques agricoles considérées comme peu esthétiques (notamment les serres). L'enjeu est de rechercher les contours d'un bien commun à l'échelle du territoire qui permettrait aux propriétaires fonciers de louer les terres agricoles dont ils disposent.

Alors que la désadaptation de l'île (Péron, 1993) avait contribué à marginaliser l'agriculture lors du second pacte territorial, elle devient désormais un creuset pour investir l'agriculture de nouvelles fonctionnalités. Le développement agricole est ainsi soutenu par une recherche d'interconnaissance et de proximité, une envie de «faire village » (Hervieu et Viard, 1996) dans le nouveau lieu de vie des néo-insulaires et sur le lieu de villégiature des résidents secondaires (Blondy et al., 2016). De plus, étant majoritairement issus de classes supérieures urbaines, ils sont sensibles à l'énonciation de leur engagement écologique (Comby, 2015). Bien que les résidents secondaires - souvent propriétaires de foncier agricole - aient été longtemps rétifs au développement de l'agriculture sur l'île, l'enjeu de la consommation locale fait évoluer leur position-

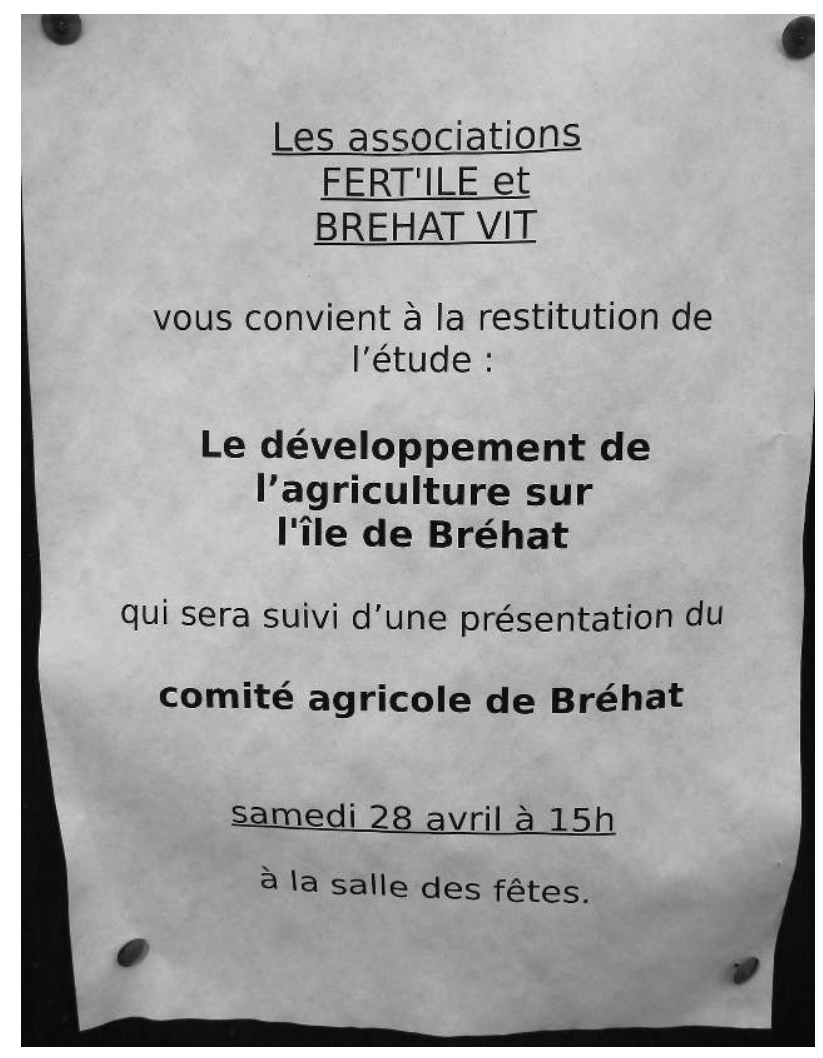

Figure 13 : Affichage annonçant une réunion publique sur la place de l'agriculture à Bréhat. Crédit photo : J. Cardinal

Poster introducing a public debate about the role of agriculture on the island. Photo credits: J. Cardinal

nement. Enfin, les agriculteurs bréhatins se voient confier un rôle de « jardiniers de la nature » (Rémy, 1990, cité par Granchamp, 2017), gardiens de la singularité paysagère de l'île, en luttant notamment contre l'enfrichement qui menace les paysages insulaires de standardisation (Brigand, 2002; Bedrani et Roche, 2017). Pour les résidents secondaires, attachés à la valeur affective du territoire, ils participent alors à garantir la transmission d'un territoire patrimonialisé.

Ces différentes fonctions associées au développement agricole ré-ouvrent des espaces de proximité, de dialogue et de négociations entre acteurs agricoles et non-agricoles, marquant le ré-ancrage de l'agriculture dans une forme de ruralité. Ces fonctions s'entrecroisent et réassemblent les trois dimensions de l'action d'Arendt (1983), séparées lors du précédent pacte qui était marqué par le repli de l'agriculture dans la sphère privée des seuls agriculteurs. Pour légitimer leur insertion sur le territoire et prétendre obtenir du foncier disponible, 
les actifs agricoles doivent s'insérer dans ce modèle d'agriculture plurifonctionnelle. L'endossement de ces nouveaux rôles est favorisé pour partie par les caractéristiques mêmes des exploitants agricoles : parmi eux, 6 se sont installés hors cadre familial, ce qui crée une rupture à la fois en termes de réseaux sociaux et familiaux, de type d'agriculture développé, et d'ancrage territorial des acteurs.

Malgré ces orientations, ce modèle d'inscription socio-territoriale des activités agricoles apparaît comme fragile, marqué par des formes de précarité économique et foncière. L'ampleur des transformations qui peuvent être réalisées par le comité agricole et la renégociation d'alliances entre résidents secondaires et permanents sont à relativiser : l'instance apparaît aujourd'hui davantage comme un espace où s'esquisse un dialogue, que comme un lieu de délibération et de régulation. L'existence de transactions foncières qui s'opèrent en dehors de son giron et la non-prise en compte de ses revendications par le conseil municipal lors de l'adoption du nouveau PLU en 2019 relativisent sa reconnaissance dans l'espace politique.

Les nouveaux espaces de proximité qu'ouvre cette ruralité sont donc différenciés. Ils ne sont pas permanents, perdent de leur dynamisme en dehors de la période estivale et bénéficient majoritairement à des usagers intermittents du territoire : alors que « les Bréhatins, eux, ils vont acheter sur le continent » (agricultrice, 2018), la démarche de consommation locale permet aux résidents secondaires de valoriser leur ancrage au territoire par leurs pratiques (Emelianoff, 2017).

Bien que ce nouveau rapprochement entre la ruralité et l'agriculture sur lîlle de Bréhat permette la renégociation publique de la place de l'agriculture sur le territoire, l'activité agricole s'apparente finalement à une prolongation des relations de service historiques qui existent sur l'île entre les résidents secondaires et touristes et les résidents permanents, y incorporant des dimensions paysagères et nourricières.

\section{Conclusion}

Après avoir constitué le socle de l'espace social local, l'activité agricole est aujourd'hui conditionnée par une économie tournée vers le tourisme et les résidents secondaires. Si au cours des dix dernières années le nombre de personnes tirant un revenu de productions agricoles a augmenté, la surface exploitée n'a pas sensiblement changé. La précarité foncière et la circulation de capitaux ont conduit à un développement d'activités agricoles qui s'affranchissent pour partie de la consommation de surfaces agricoles, les « agriculteurs sans terre » incarnant l'acmé de cette tension entre marginalisation spatiale et valorisation symbolique de l'agriculture.

La reconnaissance durable de la contribution de ces agriculteurs « jardiniers » au devenir de l'île est ainsi précaire, dans le sens où les politiques agricoles communautaires et nationales ne leur laissent que peu de place. Cette fragilité n'est pas non plus endossée politiquement par les décideurs locaux. Ce vide politique favorise le développement de modèles agricoles entrepreneuriaux variés, faiblement régulés et précaires. C'est ainsi de leur capacité à produire de nouvelles formes de ruralité, à la réinventer dans l'espace politique et social local au travers de partenariats formels et informels, que dépendent leur légitimité et la pérennité de ce nouveau pacte.

La multiplicité de stratégies d'accès au foncier déployées par les agriculteurs sont d'autant plus nécessaires que Bréhat ne dispose pas de zone rétrolittorale où les agriculteurs pourraient se replier. Les ressources de l'agriculture et les contraintes qui pèsent sur elle sont déterminées avant tout par le caractère littoral de l'île : forte attractivité, empilement de contraintes législatives limitant l'expansion agricole, socle socio-culturel favorable au maintien d'une agriculture non productiviste, etc. Si l'évolution de l'agriculture à Bréhat conduit à considérer qu'elle s'inscrit dans une nouvelle forme de ruralité, l'exacerbation des effets induits par la littoralité invite à décliner la notion d'hyper-ruralité proposée par Depraz (2017) en hyper-littoralité pour rendre compte des dynamiques socio-spatiales décrites à Bréhat, et par extension, aux territoires largement cernés par la mer.

\section{Bibliographie}

Albaladejo C., 2012. Les transformations de l'espace rural pampéen face à la mondialisation, Annales de géographie, $n^{0}$ 4, vol. 686, p. 387-409. DOI : 10.3917/ag.686.0387.

Arendt H., 1983. Condition de l'homme moderne, CalmannLévy, Paris, coll. Agora Pocket, 406 p.

Bacconnier-Baylet S., 2007. L'agriculture dans les espaces périurbains toulousains, discours, pratiques et enjeux autour de l'activité agricole dans les politiques d'aménagement, Thèse 
de géographie rurale, Université Toulouse II Le Mirail, Toulouse, $263 \mathrm{p}$.

Barbier J.-M. et Goulet F., 2013. Moins de technique, plus de nature : pour une heuristique des pratiques d'écologisation de l'agriculture, Natures Sciences Sociétés, $\mathrm{n}^{\circ}$ 2, vol. 21 , p. 200-210. DOI : 10.1051/nss/2013094.

Barthe Y., Blic D., De Heurtin J.-P., Lagneau É., Lemieux C., Linhardt D., Bellaing C.M., De Rémy C. et Trom D., 2013. Sociologie pragmatique : mode d'emploi, Politix, $n^{\circ} 3$, vol. 103, p. 175-204. DOI : 10.3917/pox.103.0173.

Bedrani N. et Roche J., 2017. Étude prospective pour le développement de l'agriculture sur l'île de Bréhat (Diagnostic agricole), Association Fert'île, Bréhat, 55 p.

Blondy N., Vacher L. et Vye D., 2016. Les résidents secondaires, des acteurs essentiels des systèmes touristiques littoraux français?, Territoire en mouvement revue de géographie et aménagement [en ligne], $\mathrm{n}^{\circ}$ 30. DOI : 10.4000/tem.3344.

Brigand L., 2002. Les îles du Ponant. Histoires et géographie des iles et archipels de la Manche et de l'Atlantique, Palantines, Quimper, $480 \mathrm{p}$.

Buнот C., 2003. Anciens et nouveaux résidents secondaires à l'île de Bréhat, Travaux de l'Institut de Géographie de Reims, $n^{\circ}$ 115, vol. 29, p. 91-105. DOI : 10.3406/tigr.2003.1465.

Buнот C., 2005. Analyse comparative de la propriété foncière. À partir des matrices cadastrales dans deux communes insulaires (île-de-Batz et Île-de-Bréhat), Norois, nº 196, p. 81-90. DOI : 10.4000/norois.427.

Canévet C., 1980. Les mutations de l'espace rural, Norois, $n^{\circ} 105$, p. 5-18.

CANÉvet C., 1992. Le modèle agricole breton: histoire et géographie d'une révolution agro-alimentaire, Thèse de doctorat, Université de Rennes 2.

Cardinal J., 2018. L'insertion territoriale de l'agriculture : mise en perspective entre l'île de Bréhat et Ploubazlanec (Côtes d'Armor), Mémoire de Master 2 Environnement, dynamiques des territoires et sociétés, AgroParisTech, non publié, $148 \mathrm{p}$.

Сомвy J.-B., 2015. À propos de la dépossession écologique des classes populaires, Savoir/Agir no 3, vol. 33, p. 23-30. DOI : 10.3917/sava.033.0023.

Corbin A., 1990. Le territoire du vide : l'Occident et le désir $d u$ rivage (1750-1840), Flammarion, Paris, coll. Champs, $407 \mathrm{p}$.

Depraz S., 2017. Penser les marges en France : l'exemple des territoires de «l'hyper-ruralité ", Bulletin de l'association des géographes français. Géographies, n 3, vol 94, p. 385-399. DOI : 10.4000/bagf.2086.

Dumont R., 1951. Voyages en France d'un agronome, M-TH, Génin, 466 p.

Emelianoff C., 2017. La fabrique territoriale des inégalités environnementales, in LARRÈre C. (dir.) Les inégalités environnementales, Paris, PUF, coll. La vie des idées, p. 82-102.

France, 1867. Vaine pâture. Société et communauté tacites. Règlements municipaux. Abandon de bestiaux attachés au piquet. Prohibition légale, Bulletin des arrêts de la Cour de cassation rendus en matière criminelle, Cour de Cassation, p. $443-452$.
Gariglietti-Brachetto C., 2013. États des lieux, dynamiques et perspectives des activités agricoles dans les îles du Ponant, Mémoire de Science de la mer et du littoral, Université de Bretagne Occidentale, $107 \mathrm{p}$.

Guillemet D., 2000. Les îles de l'Ouest : de Bréhat à Oléron, du Moyen Âge à la Révolution, Geste, La Crèche, coll. Pays d'histoire, $355 \mathrm{p}$.

Granchamp L., 2017. L'agriculture est-elle rurale ou urbaine? La mise en perspective des catégories au prisme de l'agriculture urbaine, in Hamman P. (dir.). Ruralité, nature et environnement. Entre savoirs et imaginaires, Erès, Toulouse, p. 239-274.

Hervieu B. et Hervieu-Léger D., 2005 (1979). Le retour à la Nature: au fond de la forêt, l'État, L'Aube, $2^{\mathrm{e}}$ ed., $234 \mathrm{p}$.

Hervieu B. et Purseigle F., 2013. Sociologie des mondes agricoles, Armand Colin, coll. U : Sociologie, 320 p.

Hervieu B. et Viard J., 1996. Au bonheur des campagnes (et des provinces), L'Aube, Marseille, $160 \mathrm{p}$.

Huchet du Guermeur S., 2000. "La maison de Bréhat », émergence d'un nowveau langage architectural au début $d u$ $\mathrm{XX}^{e}$ siècle, Diplôme d'architecte, École d'architecture de Nantes, 253 p.

INSEE, 2015. Statistiques locales [https://statistiques-locales. insee.fr] (consulté le 04 décembre 2019).

LAFERTÉ G., 2014. Des études rurales à l'analyse des espaces sociaux localisés, Sociologie, no 4, vol. 5, p. 423-439. DOI : 10.3917/socio.054.0423.

LaLigant S., 2008. Un point de non-retour. Anthropologie sociale d'une communauté rurale et littorale bretonne, PUR, Rennes, coll. Espaces et territoires, $394 \mathrm{p}$.

Le Pache J.-L. et Le Pache M., 1991. Bréhat : une île traverse la Révolution, autoédition, $191 \mathrm{p}$.

Marteville A. et Varin P., 1843. Dictionnaire historique et géographique de Bretagne, dédié à la Nation Bretonne. Version revue et angmentée du Dictionnaire d'Ogée, Molliex, Rennes, $538 \mathrm{p}$.

Mendras H., 1992 (1967). La fin des paysans, Actes Sud, Arles, $446 \mathrm{p}$.

Menguy L., 2005. Bribes d'histoire de l'archipel bréhatin, Association pour la sauvegarde et l'entretien du patrimoine religieux de Bréhat, $507 \mathrm{p}$.

Mormont M., 2009. Who is rural? Or how to be rural: toward a sociology of the rural, in Mardsen T.K., Lowe P., WhatMORE S., Rural restructuring: Critical perspectives on rural change, vol. 1, Fulton. 208 p.

Péron F., 2005. Fonctions sociales et dimensions subjectives des espaces insulaires (à partir de l'exemple des îles du Ponant), Annales de géographie, no 4, vol. 644, p. 422-436. DOI : 10.3917/ag.644.0422.

Péron F., 1993. Des îles et des hommes : l'insularité aujourd'hui, Éditions de la Cité, Paris ; Ouest-France, Rennes, 286 p.

Prével M., 2008. Le productivisme agricole. Socioanthropologie de l'industrialisation des campagnes françaises, Études rurales, 2008, no 181, p. 115-132. DOI : 10.4000/etudesrurales.8675. 
Rieutort L., 2009. Dynamiques rurales françaises et re-territorialisation de l'agriculture, L'information géographique, $\mathrm{n}^{\circ} 1$, vol. 73, p. 30-48. DOI : 10.3917/lig.731.0030.

Rigaud J.-M., 1890 (1995). Géographie historique des Côtes du Nord, La Tour Gile, Saint Brieuc, 2 ed., 509 p.

SALOMÉ K., 2006. Les îles bretonnes au XIX ${ }^{\mathrm{e}}$ siècle, entre éloignement et isolement, Ethnologie française, $\mathrm{n}^{\circ}$ 3, vol. 36, p. 435-441. DOI : 10.3917/ethn.063.0435.

SAntos M., 2007. O espaço do cidadão, EDUSP, São Paulo, SP, Brasil.

SEncebe Y., 2004. Être ici, être d'ici : formes d'appartenance dans le Diois (Drôme), Ethnologie française, nº 1, vol. 34, p. 23-29. DOI : 10.3917/ethn.041.0023.
Stoessel-Ritz J., 2017. Agriculture et lien social : travailler la terre et produire des transactions sociales pour le bien commun, in Hamman P. (dir.). Ruralité, nature et environnement. Entre savoirs et imaginaires, Erès, Toulouse, p. 151-179.

Talandier M., 2008. Une autre géographie du développement rural : une approche par les revenus, Géocarrefour, $\mathrm{n}^{\circ} 4$, vol. 83, p. 259-267. DOI : 10.4000/geocarrefour.7021.

Vergez-Tricom G., 1925. Lîle de Bréhat, La Géographie : bulletin de la Société de géographie, n 5, t. 43, p. 35-57.

Viart M., 2019. Projet de l'élaboration du plan local d'urbanisme de l'île de Bréhat. Rapport d'enquête (Enquête publique du 21 décembre 2018 au 22 janvier 2019), Préfecture des Côtes d'Armor, Tribunal administratif de Rennes, E 180263/35, 48 p. 